\title{
Adiabatic mean-field model for dynamical collective state transitions of a nuclear system
}

\author{
T. Kohmura, ${ }^{*}$ Y. Hashimoto, H. Ohta, and M. Maruyama ${ }^{\dagger}$ \\ Institute of Physics, University of Tsukuba, Tsukuba 305-0006, Japan \\ (Received 2 February 1999; published 18 February 2000)
}

\begin{abstract}
We propose an adiabatic mean-field model for dynamical collective state transitions of a nuclear system. The transition process is described in terms of the nuclear mean-field wave functions which are adiabatically determined in the course of the transition. A principal steering meson field approximation simplifies the model. In the simplified model, the Hamiltonian is expressed by a tridiagonal matrix on the basis of the adiabatic mean-field states, because the mean-field states are coupled by the residual interaction. The model has two degenerate lowest mean-field states. These states are separated by a potential barrier made of intermediate mean-field states and are coupled to each other by the interaction through the intermediate states. We solve the eigenvalue equation for the Hamiltonian both in an exact diagonalization and in a perturbation method. The perturbation expression for the splitting of the energies of the two almost degenerate ground states exhibits analytically a coherent structure in favor of the dynamical transition between the two isolated lowest meanfield states. The net current for the collective tunneling from an initial lowest mean-field state to the degenerate counterpart through the potential barrier is much smaller than the quantum mechanically fluctuating local currents. The energy eigenvalue equation for a tridiagonal Hamiltonian matrix leads to a Schrödinger difference equation on a finite range of integral discrete coordinates. Higher energy states on a repulsive parabolic potential on the finite range of discrete coordinate are shown to have some features resembling the energy states of a harmonic oscillator: equispacing energy levels and Gaussian distribution of the wave functions.
\end{abstract}

PACS number(s): 21.60.Ev, 24.10.Cn

\section{INTRODUCTION}

A finite many-particle system has a large number of isolated Hartree states with different symmetry. The system may make dynamical collective state transitions by tunneling from one Hartree state to another. Tunneling in manyparticle systems is an interesting problem with applications in nuclear physics to spontaneous fission as well as fusion reactions. A number of methods have been developed to treat the dynamics in the classically forbidden domain. A microscopic theory starts from a mean field or mean-field wave functions, but the residual interaction which allows the barrier penetration breaks the symmetry of these wave functions.

It has been discussed [1] that the residual interaction gives rise to the interplay between single-particle and collective motions which plays a significant role in the nuclear dynamics. The time-dependent Hartree-Fock (TDHF) calculation [2] shows that the interplay makes the nuclear system move around isolated mean-field states, i.e., Hartree minima in the quantum phase space. One of the central subjects in the TDHF theory for the nuclear collective dynamics has been to figure out the mechanism of the generation, transfiguration, and dissipation of large amplitude collective motion. The main problem is how to select a collective path in the multidimensional TDHF phase space that involves a large number of Hartree minima resulting from single-particle level crossings.

Marumori et al. have proposed a self-consistent collective

\footnotetext{
*Electronic address: kohmura@nucl.ph.tsukuba.ac.jp

†Permanent address: Department of Physics, Tohoku University, Sendai 980-8578, Japan.
}

coordinate (SCC) method to select the "optimum', collective path in the TDHF phase space. This method uses the power series expansion in terms of dynamical collective variables defined at a Hartree minimum. We select the collective subspace in the phase space where solution of the nonlinear TDHF equation evolves. This is an extension of the random phase approximation (RPA) toward the problems of large amplitude collective motion [1]. The method in fact has been successfully applied to realistic problems to study the microscopic mechanism of anharmonic $\gamma$ vibrations [3] and of band-crossing phenomena in nuclear rotational motions [4].

The power series expansion in terms of the collective variables in the SCC method is defined in the space of oneSlater-determinant wave function. This is not applicable to nuclear problems of the barrier penetration for collective motion. In order to extend the applicability of the method toward the problems of barrier penetration, we shall introduce a new theoretical framework based on the adiabatic meanfield theory. The expression for nuclear collective state transitions in terms of adiabatic mean-field wave functions representing a number of Slater determinants is exploited to incorporate appropriately the effects of the single-particle level crossings in the transition process.

Before starting the description of the adiabatic mean-field model, we refer to Arve et al. who proposed a simple model for tunneling in many-particle systems to test various methods of treating large amplitude collective motion [5]. To provide a model of barrier penetration, the energies of the Hartree states are low in two distinct regions of configuration space. These are separated by a barrier region with high Hartree energies, so that tunneling physics applies to the low eigenstates. The Hamiltonian governing the dynamics of $N$ distinguishable particles has a residual interaction that breaks 
the Hartree symmetries and allows the wave function to spread over the entire configuration space. Each of the particles has a $z$ coordinate as well as an internal spin coordinate. The Hamiltonian reads

$$
\begin{aligned}
H= & \sum_{i=1}^{N} \frac{1}{2}\left(-\frac{d^{2}}{d z_{i}^{2}}+z_{i}^{2}\right)+\kappa\left(\sum_{i=1}^{N} z_{i}\right)\left\{\sum_{i=1}^{N} \sigma_{z}(i)\right\} \\
& +\lambda\left\{\sum_{i=1}^{N} \sigma_{x}(i)\right\}^{2} .
\end{aligned}
$$

The first two terms represent a Hartree Hamiltonian and the last term is a residual interaction. Their goal of the treatments of the Hamiltonian is to reproduce the degeneracy splitting of states of opposite parity with respect to the center of the barrier and to compare the results of several methods.

They first diagonalize the Hamiltonian matrix in a space truncated to limited oscillator states with the parameters physically chosen. The accuracy of the splittings and average energies in the truncation to the lowest oscillator state $\nu=0$ shows that the single-particle oscillator motion is irrelevant for the tunneling dynamics. They next obtain eigenfunctions of the constrained mean-field Hamiltonian in both the $\langle z\rangle$ and $\left\langle\sigma_{z}\right\rangle$ constrained mean-field approximations. The constrained Hartree calculations indicate that one should not make an a priori choice of collective path but rather use a theory that selects the optimum path for the process under consideration. They then calculate the splitting of the degeneracy, solving the imaginary time mean-field equation and also using the continuum hopping model. It is made clear that different schemes supplement each other in having different domains of validity.

We finally quote their following statement [5]: "The dynamics in the barrier are governed by changing the spin occupation, which corresponds to level crossings of singleparticle states of different nodal structure in the physical problem. Hence constraining the spin occupation directly gives a better approximation than influencing it only indirectly through constraining the collective variable.'

Extending their intuitive model, we develop a fundamental and applicable description for nuclear collective transitions between Hartree minima in terms of the adiabatic mean-field approximation. In the previous papers [6], we solved the mean-field problems for static properties of nuclei. There is a nucleus presumably composed of a number of nucleons and several kinds of mesons. We express the metastable states of the nucleus in terms of the mean-field approximation. The mesons produce their mean fields to hold the mean-field (Hartree) states of the nucleons. We expand the meson fields in the nucleus on oscillator basis.

Presently, extending the mean-field treatment of a nucleus to the dynamical problems, we propose an adiabatic meanfield model for nuclear collective state transitions. A nuclear dynamical transition from one metastable state to another is formulated in the steering meson field approximation. In the course of the nuclear transition, we assume that each of the participant nucleons changes the single-particle state at a level crossing, while the system traverses the potential barrier [7]. The nuclear transition is completed when all of the participant nucleons finish changing their single-particle states. Therefore, in order to describe the collective state transitions, it is significant to take into account all of the participant nucleons.

In the adiabatic mean-field model, the nuclear transition process is described in terms of the mean-field wave functions which are adiabatically determined, while the nucleon configuration changes itself step by step in the course of the nuclear transition. The changing nucleon configuration varies the meson mean fields. The varying meson mean fields shift in turn the energy levels of single-particle states for each participant nucleon to cross each other. Thus the nucleon configuration changes in accordance with the meson mean fields in the transition process.

In the present paper, we take the principal steering meson field approximation, a simplified version of the adiabatic mean-field model, since this visualizes the fundamental and typical features of dynamical collective state transitions between isolated Hartree minima. In the approximation, we take only one principal meson field to steer the nuclear transition and assign two energy levels for each of the participant nucleons to change its single-particle state once in the transition process: The two single-particle states for each of the nucleons are labeled as states $s=1$ and -1 . The singleparticle energies are adiabatically determined by the meson mean field steering the nuclear transition, so that the two single-particle energy levels of the nucleons cross each other.

We make the present model system have two degenerate lowest mean-field states (Hartree minima) separated by a potential barrier with high mean-field energies of intermediate states. The model Hamiltonian is expressed by a tridiagonal matrix on the basis of the adiabatic mean-field states, because the mean-field states are coupled by the residual interaction. The two degenerate lowest mean-field states are coupled to each other by high orders of the residual interaction through the intermediate states. The coupling of the two degenerate states splits the degeneracy.

The Hamiltonian in the present simplified version of the adiabatic mean-field model has mathematically a same form as that in Eq. (1) used for the harmonic oscillator coupled to the spin for nucleons in Ref. [5]. Since our Hamiltonian is formulated in terms of meson theory, this formulation is more fundamental and applicable than the intuitive model of Arve et al.

While one usually calculates the splitting of the energies of the two almost degenerate ground states in the WKB method using imaginary time [8], we obtain the degeneracy splitting both in an exact diagonalization and in a perturbation method. We discuss the fact that the perturbation calculation works better for the present problem than the WKB method.

Here, we remark on some aims of the present work, which is to discuss the features of the nuclear transitions between mean-field minima. The energy eigenvalue equation for a tridiagonal mean-field Hamiltonian matrix leads to a Schrödinger difference equation on a finite range of integral discrete coordinates. The first aim is to show that higher energy states on the repulsive parabolic potential on the finite range of discrete coordinates have some features resembling the 
energy states of a harmonic oscillator: equispacing energy levels and Gaussian distribution of the wave functions.

We calculate the nuclear transition probability from one of the two degenerate lowest mean-field states to the degenerate counterpart. The second aim is to show that the net current for the collective tunneling through the potential barrier is typically small compared with quantum mechanically fluctuating local currents: the transition probability oscillates in the two mixed modes of tunneling and of quantum fluctuation.

The tridiagonal mean-field Hamiltonian matrix has a coherent structure in favor of dynamical collective state transitions. The third aim is to discuss that the adiabatic mean-field Hamiltonian matrix for physical problems in a more general case may be transformed into a tridiagonal matrix, which selects the optimum collective transition path between Hartree minima with the transformation matrix providing the path.

In Sec. II, the principal steering meson field approximation of the adiabatic mean-field model is described for dynamical collective state transitions between isolated Hartree minima. In Sec. III, we solve the eigenvalue equation for the Hamiltonian on the basis of the adiabatic mean-field states. In Sec. IV, we solve the evolution problem for the dynamical collective state transitions of the nuclear system. Conclusions and discussion are given in Sec. V.

\section{ADIABATIC MEAN-FIELD MODEL}

\section{A. Principal steering meson field approximation}

The relativistic mean-field theory of a nucleus has been successfully applied to describe static properties of nuclei [9]. A nucleus is presumably composed of a number of nucleons and some kinds of mesons. In this paper we start with the relativistic mean-field expression for a nucleus in terms of the nucleon field $\psi$ and scalar meson field $\phi$, where we do not express explicitly other meson degrees of freedom, for simplicity.

The Hamiltonian for the system reads

$$
\begin{aligned}
H= & \int\left\{\bar{\psi}(\vec{\gamma} \cdot \vec{\nabla}+M) \psi-g \bar{\psi} \psi \phi+\frac{1}{2}\left(\Pi^{2}+\nabla \phi \cdot \nabla \phi\right.\right. \\
& \left.\left.+m^{2} \phi^{2}\right)\right\} d^{3} r,
\end{aligned}
$$

with the momentum field $\Pi=\dot{\phi}$ for the meson. The quantum fields satisfy the equation of motion,

$$
\begin{aligned}
& \left(\frac{\partial^{2}}{\partial t^{2}}-\nabla^{2}+m^{2}\right) \phi=g \bar{\psi} \psi, \\
& \left(\gamma_{\mu} \frac{\partial}{\partial x_{\mu}}+M-g \phi\right) \psi=0 .
\end{aligned}
$$

The meson field $\phi$ in a finite nuclear system is expanded on oscillator basis $\varphi_{n l m}[6]$,

$$
\phi(\vec{r})=\sum_{n l m}\left\{a_{n l m} \varphi_{n l m}(\vec{r})+a_{n l m}^{\dagger} \varphi_{n l m}^{*}(\vec{r})\right\}
$$

with the annihilation and creation operators $a_{n l m}$ and $a_{n l m}^{\dagger}$, respectively.

To describe a dynamical collective state transition between Hartree minima with different symmetries, we first solve the static nuclear problems for the initial and final Hartree states in the mean-field approximation. A metastable nuclear state is expressed as

$$
|\Psi\rangle=\left|\Psi_{0}\right\rangle *\left|\Phi_{0}\right\rangle,
$$

with a coherent state of mesons,

$$
\left|\Phi_{0}\right\rangle=\prod_{n l m}\left(e^{\alpha_{n l m} a_{n l m}^{\dagger}} e^{-\left|\alpha_{n l m}\right|^{2} / 2}\right)|0\rangle,
$$

and a Slater determinant state of the nucleons:

$$
\left|\Psi_{0}\right\rangle=c^{0 \dagger}{ }_{j_{1}}^{\dagger} \cdots c_{j_{N}}^{0 \dagger}|0\rangle .
$$

In the mean-field approximation, both quantum fields $\bar{\psi} \psi$ and $\phi$ are expressed by a sum of the mean field and quantum fluctuation,

$$
\begin{gathered}
\left(\frac{\partial^{2}}{\partial t^{2}}-\nabla^{2}+m^{2}\right)(\langle\phi\rangle+\hat{\phi})=g(\langle\bar{\psi} \psi\rangle+: \bar{\psi} \\
\left\{\gamma_{\mu} \frac{\partial}{\partial x_{\mu}}+M-g(\langle\phi\rangle+\hat{\phi})\right\} \psi=0,
\end{gathered}
$$

where the mean fields are defined to be the expectation value of the fields in the nuclear state,

$$
\begin{gathered}
\langle\phi\rangle=\left\langle\Phi_{0}|\phi(\vec{r})| \Phi_{0}\right\rangle=\varphi_{0}(\vec{r}), \\
\langle\bar{\psi} \psi\rangle=\left\langle\Psi_{0}|\bar{\psi}(\vec{r}) \psi(\vec{r})| \Psi_{0}\right\rangle=\sum_{j=j_{1}}^{j_{N}} \bar{\psi}_{j}^{0}(\vec{r}) \psi_{j}^{0}(\vec{r}) .
\end{gathered}
$$

Solving the simultaneous mean-field equations

$$
\begin{aligned}
& \left(-\nabla^{2}+m^{2}\right) \varphi_{0}(\vec{r})=g \sum_{j=j_{1}}^{j_{N}} \bar{\psi}_{j}^{0}(\vec{r}) \psi_{j}^{0}(\vec{r}), \\
& \beta\left\{\vec{\gamma} \cdot \vec{\nabla}+M-g \varphi_{0}(\vec{r})\right\} \psi_{j}^{0}(\vec{r})=\varepsilon_{j} \psi_{j}^{0}(\vec{r}),
\end{aligned}
$$

with the meson mean field expanded on an oscillator basis,

$$
\varphi_{0}(\vec{r})=\sum_{n l m}\left\{\alpha_{n l m} \varphi_{n l m}(\vec{r})+\alpha_{n l m}^{*} \varphi_{n l m}^{*}(\vec{r})\right\}
$$

we obtain the mean-field expression $|\Psi\rangle=\left|\Psi_{0}\right\rangle *\left|\Phi_{0}\right\rangle$ for the static nuclear state.

We now turn to a dynamical collective state transition from the initial nuclear mean-field state $\Psi_{\mathrm{i}}$ to the final $\Psi_{\mathrm{f}}$, for which we have solved the static mean-field equations. The two nuclear mean-field states $\Psi_{\mathrm{i}}$ and $\Psi_{\mathrm{f}}$ are specified by the mean field values $\alpha_{n l m}^{\mathrm{i}}$ and $\alpha_{n l m}^{\mathrm{f}}$, respectively, of the 
meson operators $a_{n l m}$. In the principal steering meson field approximation for the dynamical collective state transition between these states, we assume that the transition proceeds with the meson mean fields $\alpha_{n l m}$ varying along a straight line,

$$
\alpha_{n l m}(x)=(1-x) \alpha_{n l m}^{\mathrm{i}}+x \alpha_{n l m}^{\mathrm{f}},
$$

in the range $0 \leqslant x \leqslant 1$, starting with $x=0$ for $\Psi_{\mathrm{i}}$ and terminating at $x=1$ for $\Psi_{\mathrm{f}}$.

We can obtain the single-particle states of the nucleons in the meson mean fields $\alpha_{n l m}(x)$ at given $x$. When the parameter $x$ is varied, the single-particle levels of valence nucleons shift to cross each other. This assigns two single-particle states for each of the participant nucleons to change the state while the nuclear system makes a transition from the initial state $\Psi_{\mathrm{i}}$ to the final $\Psi_{\mathrm{f}}$. We label the two single-particle states as $s=1$ and -1 for each of the participant nucleons. The participant nucleons occupy the single-particle state $s$ $=-1$ in the nuclear mean-field state $\Psi_{\mathrm{i}}$ and the state $s=1$ in the state $\Psi_{\mathrm{f}}$. They change the single-particle state while the meson mean fields vary from the initial values $\alpha_{n l m}^{\mathrm{i}}$ to the final $\alpha_{n l m}^{\mathrm{f}}$ in the transition process. The energies of the two single-particle states are adiabatically determined by the meson mean field in the course of the nuclear transition, so that the two single-particle energy levels of the nucleons cross each other. Each nucleon changes the single-particle state at the level crossing.

Using the mean field $\varphi_{0}^{\mathrm{i}}(\vec{r})$ in the nuclear state $\Psi_{\mathrm{i}}$, we express the meson field operator as

$$
\phi(\vec{r})=\varphi_{0}^{\mathrm{i}}(\vec{r})+\hat{\phi}(\vec{r}),
$$

where the mean field is expanded on an oscillator basis,

$$
\varphi_{0}^{\mathrm{i}}(\vec{r})=\sum_{n l m}\left\{\alpha_{n l m}^{\mathrm{i}} \varphi_{n l m}(\vec{r})+\alpha_{n l m}^{\mathrm{i} *} \varphi_{n l m}^{*}(\vec{r})\right\}
$$

In order to quantize the meson fluctuation field $\hat{\phi}(\vec{r})$, we define the principal meson field to steer the nuclear transition,

$$
\varphi_{1}(\vec{r})=N_{1} \sum_{n l m}\left(\alpha_{n l m}^{\mathrm{f}}-\alpha_{n l m}^{\mathrm{i}}\right) \varphi_{n l m}(\vec{r}),
$$

with a normalization factor $N_{1}$, which expresses the meson field $\alpha_{n l m}$, varying from $\alpha_{n l m}^{\mathrm{i}}$ to $\alpha_{n l m}^{\mathrm{f}}$ in the course of nuclear transition. The other orthogonal basis functions are defined to be

$$
\varphi_{k}(\vec{r})=N_{k} \sum_{n l m}\left(\alpha_{n l m}^{k}-\alpha_{n l m}^{\mathrm{i}}\right) \varphi_{n l m}(\vec{r}),
$$

where the vector $\alpha_{n l m}^{\mathrm{f}}-\alpha_{n l m}^{\mathrm{i}}$ for the principal steering field and the other vectors $\alpha_{n l m}^{k}-\alpha_{n l m}^{\mathrm{i}}$ for $k=2,3, \ldots$ are assumed to be orthogonal to each other in the meson meanfield $\alpha_{n l m}$ space. On the basis of the functions $\varphi_{k}(\vec{r})$ for $k$ $=1,2, \ldots$, we quantize the meson quantum fluctuation field in Eq. (16) as

$$
\hat{\phi}(\vec{r})=\sum_{k=1}\left\{a_{k} \varphi_{k}(\vec{r})+a_{k}^{\dagger} \varphi_{k}^{*}(\vec{r})\right\}
$$

with the operators $a_{k}$ and $a_{k}^{\dagger}$. The residual interaction which motivates the nuclear transition from the state $\Psi_{\mathrm{i}}$ to $\Psi_{\mathrm{f}}$ is described by the nucleon-nucleon interaction exchanging one meson in the principal steering state $\varphi_{1}(\vec{r})$. The principal steering meson field may be a linear combination of scalar and vector meson fields in the $\sigma$ and $\omega$ meson mean-field approximation for the nucleus. We discuss further the steering meson field approximation in the relativistic mean-field theory in Sec. V.

In the principal steering meson field approximation of the adiabatic mean-field model, we take into account only the steering meson field $\varphi_{1}(\vec{r})$ in the fluctuation field in Eq. (19). A more general treatment to take into account other fields with $k=2,3, \ldots$ in the fluctuation field is also discussed in Sec. V.

We take a nuclear system which is composed of $N$ nucleons $(j=1, \ldots, N)$, interacting with the mesons occupying the steering state $\varphi_{1}(\vec{r})$ in Eq. (17). The nucleons occupy one of their two individual states $(s=1,-1)$. The Hamiltonian for the system is reduced to

$$
\begin{gathered}
H=E\left(\alpha_{n l m}^{\mathrm{i}}\right)+\frac{\left(g_{1} N\right)^{2}}{\varepsilon}+H_{0}+H_{I}, \\
H_{0}=\varepsilon a^{\dagger} a-g_{1} \sum_{j s} s c_{j s}^{\dagger} c_{j s}\left(a+a^{\dagger}\right), \\
H_{I}=-\frac{g_{2}^{2}}{2 \varepsilon} \sum_{i j}\left(c_{i 1}^{\dagger} c_{i-1}+c_{i-1}^{\dagger} c_{i 1}\right)\left(c_{j 1}^{\dagger} c_{j-1}+c_{j-1}^{\dagger} c_{j 1}\right),
\end{gathered}
$$

where the operators $c_{j s}$ and $c_{j s}^{\dagger}$ are for the nucleons $j$ $=1,2, \ldots, N$ in the states $s=-1,1$ and the operators $a$ and $a^{\dagger}$ stand for $a_{1}$ and $a_{1}^{\dagger}$ for the steering field $\varphi_{1}(\vec{r})$, respectively. The energy $E\left(\alpha_{n l m}^{\mathrm{i}}\right)$ is the nuclear mean-field enegy for the state $\Psi_{\mathrm{i}}$ and the energy correction $\left(g_{1} N\right)^{2} / \varepsilon$ is to cancel the mean-field energy from $H_{0}$. The Hamiltonian $H_{0}$ provides the meson mean field varying with nucleon configurations and $H_{I}$ is for the residual interaction exchanging one meson in the state $\varphi_{1}(\vec{r})$ to steer the nuclear collective state transitions between the mean-field minima. We assume that the two lowest mean-field states are degenerate.

The present Hamiltonian $H_{0}+H_{I}$ has the same form mathematically as that in Eq. (1) used in Ref. [5]. The authors of Ref. [5] choose the parameters for their Hamiltonian appropriate to the nuclear tunneling in spontaneous fission. They choose $N=40$ for the number of level crossing below the barrier. For the energy of collective single-particle motion, they identify the single-particle frequency with the giant quadrupole vibration, which is of the order of $10-15 \mathrm{MeV}$. A typical value for the barrier height for spontaneous fission from the ground state is $5 \mathrm{MeV}$. The matrix element for the residual interaction connecting adjacent configurations is of the order of magnitude of $2-3 \mathrm{MeV}$. In the numerical calcu- 
lations in the present work, we use the set of parameters $g_{1}$ $=0.006403 \sqrt{20}, g_{2}=\sqrt{0.001}$, and $\varepsilon=1$ in the unit scale of the order of magnitude of $10-15 \mathrm{MeV}$ and $N=40$, which are adjusted to reproduce the parameters that in Ref. [5] are chosen from the above considerations, for comparison's sake. The values of the parameters derived from the relativistic mean-field approximation are discussed in Sec. V.

\section{B. Adiabatic mean-field approximation}

Now we apply the adiabatic meson mean-field approximation to the Hamiltonian $H_{0}$. The nuclear system of $N$ nucleons is described in terms of the symmetric nucleon configurations

$$
|\Psi(n, N-n)\rangle=\frac{1}{\sqrt{{ }_{N} C_{n}}} \sum_{P} P c_{11}^{\dagger} \cdots c_{n 1}^{\dagger} c_{n+1-1}^{\dagger} \cdots c_{N-1}^{\dagger}|0\rangle,
$$

where $n$ nucleons occupy the single-particle state $s=1$ and the other $N-n$ nucleons the state $s=-1$. The permutations $P$ stand for those between some of the nucleons $j$ $=1, \ldots, n$ occupying the state $s=1$ and the counterpart of the nucleons $j=n+1, \ldots, N$ occupying the state $s=-1$.

The Hamiltonian $H_{0}$ is diagonal on the basis of the nucleon configurations $\Psi(n, N-n)$ so that these nuclear states are the eigenstates of $H_{0}$ in the meson mean-field approximation. In the approximation, the meson states are described by a coherent state [10]

$$
|\Phi(\alpha)\rangle=e^{\alpha a^{\dagger}}|0\rangle / e^{|\alpha|^{2} / 2},
$$

which is an eigenstate of the annihilation operator $a$, satisfying the eigenequation

$$
a|\Phi(\alpha)\rangle=\alpha|\Phi(\alpha)\rangle
$$

with its conjugate equation

$$
\langle\Phi(\alpha)| a^{\dagger}=\alpha^{*}\langle\Phi(\alpha)| .
$$

We assume that the meson coherent state takes a real value of the mean-field parameter $\alpha$. The parameter $\alpha$ for the coherent state reproduces the expectation (mean-field) value of the meson operators $a$ and $a^{\dagger}$ in the nuclear state,

$$
\alpha=\langle\Phi(\alpha)|a| \Phi(\alpha)\rangle=\left\langle\Phi(\alpha)\left|a^{\dagger}\right| \Phi(\alpha)\right\rangle .
$$

In terms of nucleon configuration $\Psi(n, N-n)$ and meson coherent state $\Phi(\alpha)$, we express the nuclear mean-field states as

$$
|\check{\Psi}\rangle=|\Psi(n, N-n)\rangle \cdot|\Phi(\alpha)\rangle .
$$

The nuclear mean-field energy for the state $|\check{\Psi}\rangle$ depends on the nucleon configuration $\Psi(n, N-n)$ and the meson mean field $\alpha$,

$$
\begin{aligned}
E(n, \alpha) & =\left\langle\check{\Psi}\left|H_{0}\right| \check{\Psi}\right\rangle=\varepsilon \alpha^{2}-2 g_{1}(2 n-N) \alpha \\
& =\varepsilon\left\{\alpha-\frac{g_{1}}{\varepsilon}(2 n-N)\right\}^{2}-\frac{g_{1}^{2}}{\varepsilon}(2 n-N)^{2},
\end{aligned}
$$

where we have substituted the expectation value of the bilinear nucleon operator,

$$
\left\langle\Psi(n, N-n)\left|\sum_{j s} s c_{j s}^{\dagger} c_{j s}\right| \Psi(n, N-n)\right\rangle=2 n-N .
$$

Therefore, for each of the nucleon configurations $\Psi(n, N$ $-n)$, the meson mean field

$$
\alpha=\alpha_{n}=\frac{g_{1}}{\varepsilon}(2 n-N)
$$

is adiabatically determined to minimize the nuclear meanfield energy $E(n, \alpha)$. Substituting this value $\alpha_{n}$ of $\alpha$ into the nuclear mean-field state in Eq. (28) and into the nuclear mean-field energy $E(n, \alpha)$, we obtain the adiabatic nuclear mean-field state

$$
|\check{\Psi}(n)\rangle=|\Psi(n, N-n)\rangle \cdot\left|\Phi\left(\alpha_{n}\right)\right\rangle
$$

and the adiabatic energy

$$
\varepsilon_{n}=E\left(n, \alpha_{n}\right)=-\frac{g_{1}^{2}}{\varepsilon}(2 n-N)^{2},
$$

respectively, for each of the nucleon configurations $|\Psi(n, N-n)\rangle$.

The present nuclear system has two ground states in the adiabatic meson mean-field approximation for $H_{0}$ : one is the nuclear mean-field state $\check{\Psi}(0)$ and the other is $\check{\Psi}(N)$, whose energies are degenerate:

$$
\varepsilon_{0}=\varepsilon_{N}=-\frac{g_{1}^{2}}{\varepsilon} N^{2} .
$$

The two degenerate lowest mean-field states $\check{\Psi}(0)$ and $\check{\Psi}(N)$ are coupled to each other by the residual interaction in the Hamiltonian $H_{I}$ through intermediate states $\check{\Psi}(n)$ and make the two nuclear ground state energies slightly split in the case of a nuclear system of an even number $N$ of nucleons.

On the basis of the adiabatic mean-field states, we solve the energy eigenvalue equation for the Hamiltonian $H_{0}$ $+H_{I}$ in Sec. III and calculate the transition probabilities between the adiabatic mean-field states in Sec. IV.

\section{ENERGY EIGENSTATES FOR ADIABATIC MEAN-FIELD HAMILTONIAN}

\section{A. Diagonalization of the Hamiltonian matrix}

In a nuclear system of an even number $N$ of nucleons, the $N / 2+1$ adiabatic nuclear mean-field states 
$\check{\Psi}(0), \check{\Psi}(2), \ldots, \check{\Psi}(N)$ are coupled by the residual interaction. Using these adiabatic mean-field states $\check{\Psi}(n)$ for basis states, we make an $N / 2+1$ by $N / 2+1$ tridiagonal matrix expression for the Hamiltonian $H=H_{0}+H_{I}$,

$$
H=\left(\begin{array}{ccccccc}
\tilde{\varepsilon}_{0} & h_{02} & & & & & 0 \\
h_{20} & \tilde{\varepsilon}_{2} & h_{24} & & & & \\
& h_{42} & \tilde{\varepsilon}_{4} & h_{46} & & & \\
& & & \ldots & & & \\
& & & & h_{N-2 N-4} & \tilde{\varepsilon}_{N-2} & h_{N-2 N} \\
0 & & & & & h_{N N-2} & \tilde{\varepsilon}_{N}
\end{array}\right)
$$

The diagonal matrix elements are

$$
\begin{aligned}
\tilde{\varepsilon}_{n}=\varepsilon_{n}+h_{n n} & =\left\langle\check{\Psi}(n)\left|\left(H_{0}+H_{I}\right)\right| \check{\Psi}(n)\right\rangle \\
& =-\frac{g_{1}^{2}}{\varepsilon}(2 n-N)^{2}-\frac{g_{2}^{2}}{\varepsilon}\left\{n(N-n)+\frac{N}{2}\right\},
\end{aligned}
$$

and the nondiagonal elements are

$$
\begin{aligned}
h_{n-2 n}=h_{n n-2} & =\left\langle\check{\Psi}(n)\left|H_{I}\right| \check{\Psi}(n-2)\right\rangle \\
& =-{ }_{n} \mathrm{C}_{2} \sqrt{\frac{{ }_{N} \mathrm{C}_{n}}{{ }_{N} \mathrm{C}_{n-2}}} \frac{g_{2}^{2}}{\varepsilon} e^{-8 g_{1}^{2} / \varepsilon^{2}} .
\end{aligned}
$$

The factor $e^{-8 g_{1}^{2} / \varepsilon^{2}}$ in the nondiagonal elements comes from the overlap integral between the meson mean-field states,

$$
\begin{aligned}
\left\langle\Phi\left(\alpha_{n-2}\right) \mid \Phi\left(\alpha_{n}\right)\right\rangle & =\left\langle\Phi\left(\alpha_{n}\right) \mid \Phi\left(\alpha_{n-2}\right)\right\rangle=e^{-\left(\alpha_{n}-\alpha_{n-2}\right)^{2} / 2} \\
& =e^{-8 g_{1}^{2} / \varepsilon^{2}}
\end{aligned}
$$

The other nondiagonal elements vanish. This adiabatic mean-field Hamiltonian matrix $H$ is characterized by the reflection symmetries $\tilde{\varepsilon}_{n}=\widetilde{\varepsilon}_{N-n}, \quad h_{n-2 n}=h_{n n-2}$ $=h_{N-n+2 N-n}=h_{N-n N-n+2}$.

We solve numerically the energy eigenvalue equation $H \check{\Psi}_{k}=E_{k} \check{\Psi}_{k}$ using the set of the parameters shown in Sec. II, which are adjusted to reproduce the parameters used for their Hamiltonian in Ref. [5]. We show the calculated nuclear eigenenergies $E_{k}$ for the Hamiltonian matrix $H$ in Table I and the calculated coefficients $c_{2 n}$ of the nuclear energy eigenstates $\check{\Psi}_{k}=\Sigma_{n} c_{2 n} \check{\Psi}(2 n)$ expanded in terms of the adiabatic mean-field states $\check{\Psi}(2 n)$ in Table II.

Note that the pair of nuclear mean-field states $\check{\Psi}(n)$ and $\check{\Psi}(N-n)$ are degenerate in the adiabatic meson mean-field approximation for the Hamiltonian $H_{0}$ and that they are coupled to each other by high orders of the residual interaction in $H_{I}$, which makes a splitting of the two concerned nuclear eigenenergies. However, since a pair of adiabatic mean-field states $\check{\Psi}(n)$ and $\check{\Psi}(N-n)$ with smaller $n$ are
TABLE I. The calculated energy eigenvalues $E_{k}$ for the Hamiltonian $H=H_{0}+H_{I}$. In the calculation, we use the set of the parameters defined in the text (see Sec. II).

\begin{tabular}{cc}
\hline \hline$k$ & $E_{k}$ \\
\hline 20 & -1.33662612166792 \\
19 & -1.33662612166779 \\
18 & -1.18228737438742 \\
17 & -1.18228737312892 \\
16 & -1.04733247693704 \\
15 & -1.04733115893980 \\
14 & -0.93395197245745 \\
13 & -0.93364759115588 \\
12 & -0.85329429700537 \\
11 & -0.84130974773678 \\
10 & -0.79386466621058 \\
9 & -0.74954840522423 \\
8 & -0.69543553240875 \\
7 & -0.63510081460674 \\
6 & -0.56871237327018 \\
5 & -0.49656399116752 \\
4 & -0.41882154872403 \\
3 & -0.33559694990056 \\
2 & -0.24696826771199 \\
1 & -0.15299183527178 \\
0 & -0.05370935801928 \\
\hline \hline
\end{tabular}

more indirectly coupled to each other by the interaction through more intermediate mean-field states, the splitting of the two nuclear energy eigenvalues is smaller. The two lowest mean-field states $\check{\Psi}(0)$ and $\check{\Psi}(N)$, which are degenerate in the mean-field approximation, are coupled to each other through the largest number of intermediate mean-field states $\check{\Psi}(2 n)$ to yield a very slight splitting of the energies of the two almost degenerate nuclear ground states. In the present numerical calculation, the two almost degenerate ground state energies $E_{19}$ and $E_{20}$ are split as slightly as by the order of $1.29 \times 10^{-13}$ as is seen in Table I.

The two almost degenerate ground states are mainly of a symmetric and antisymmetric linear combination of the two degenerate lowest mean-field basis states $\check{\Psi}(0)$ and $\check{\Psi}(N)$. These two mean-field states are coupled to each other through the intermediate states $\check{\Psi}(2 n)$ to make a complete mixing of themselves. This is analytically shown in the perturbation theory in the following subsection.

The energy eigenvalue equation $H \check{\Psi}_{k}=E_{k} \check{\Psi}_{k}$ for the tridiagonal Hamiltonian matrix $H$ leads to a Schrödinger difference equation [5] on the finite range of discrete coordinate of even integral numbers $2 n$ from 0 to $N$,

$$
\begin{aligned}
E_{k} c_{2 n} & =\varepsilon_{2 n} c_{2 n}+\sum_{m} h_{2 n 2 m} c_{2 m} \\
& =\frac{\Delta}{\Delta n} h_{2 n} \frac{\Delta}{\Delta n} c_{2 n}+\left(\tilde{\varepsilon}_{2 n}+h_{2 n 2 n-2}+h_{2 n 2 n+2}\right) c_{2 n},
\end{aligned}
$$


TABLE II. The expansion coefficients $c_{2 n}$ of the nuclear energy eigenstates $\check{\Psi}_{k}=\Sigma_{n} c_{2 n} \check{\Psi}(2 n)$ in terms of the adiabatic mean-field states $\check{\Psi}(2 n)$, which are calculated in the diagonalization method. The upper and lower signs in the double signs are for the smaller and larger of the two $2 n$ 's, respectively. The ellipses indicate a value smaller than 0.001 . The parameters used in this calculation are shown in the text.

\begin{tabular}{|c|c|c|c|c|c|c|c|}
\hline $2 n \backslash k$ & 20 & 19 & 18 & 17 & 16 & 15 & 14 \\
\hline 0,40 & 0.697 & $\mp 0.697$ & -0.117 & \pm 0.117 & 0.027 & $\mp 0.027$ & -0.007 \\
\hline 2,38 & 0.117 & $\mp 0.117$ & 0.630 & $\mp 0.630$ & -0.275 & \pm 0.275 & 0.106 \\
\hline 4,36 & 0.024 & $\mp 0.024$ & 0.281 & $\mp 0.281$ & 0.463 & $\mp 0.463$ & -0.366 \\
\hline 6,34 & 0.005 & $\mp 0.005$ & 0.098 & $\mp 0.098$ & 0.393 & $\mp 0.393$ & 0.193 \\
\hline 8,32 & 0.001 & $\mp 0.001$ & 0.032 & $\mp 0.032$ & 0.211 & $\mp 0.211$ & 0.388 \\
\hline 10,30 & $\ldots$ & $\ldots$ & 0.010 & $\mp 0.010$ & 0.095 & $\mp 0.095$ & 0.323 \\
\hline 12,28 & $\cdots$ & $\cdots$ & 0.003 & $\mp 0.003$ & 0.039 & $\mp 0.039$ & 0.207 \\
\hline 14,26 & $\cdots$ & $\cdots$ & 0.001 & $\mp 0.001$ & 0.015 & $\mp 0.015$ & 0.118 \\
\hline 16,24 & $\ldots$ & $\ldots$ & $\ldots$ & $\ldots$ & 0.006 & $\mp 0.006$ & 0.065 \\
\hline 18,22 & $\cdots$ & $\ldots$ & $\cdots$ & $\ldots$ & 0.003 & $\mp 0.002$ & 0.040 \\
\hline 20 & $\cdots$ & $\cdots$ & $\cdots$ & $\cdots$ & 0.002 & 0.000 & 0.032 \\
\hline $2 n \backslash k$ & 13 & 12 & 11 & 10 & 9 & 8 & 7 \\
\hline 0,40 & \pm 0.007 & 0.002 & $\mp 0.003$ & -0.001 & $\mp 0.001$ & 0.001 & $\ldots$ \\
\hline 2,38 & $\mp 0.106$ & -0.041 & \pm 0.045 & 0.028 & \pm 0.021 & -0.013 & \pm 0.008 \\
\hline 4,36 & \pm 0.368 & 0.192 & $\mp 0.220$ & -0.158 & $\mp 0.135$ & 0.096 & $\mp 0.063$ \\
\hline 6,34 & $\mp 0.196$ & -0.272 & \pm 0.341 & 0.325 & \pm 0.340 & -0.298 & \pm 0.235 \\
\hline 8,32 & $\mp 0.390$ & -0.120 & \pm 0.101 & -0.068 & $\mp 0.216$ & 0.336 & $\mp 0.388$ \\
\hline 10,30 & $\mp 0.323$ & 0.144 & $\mp 0.222$ & -0.293 & $\mp 0.287$ & 0.131 & \pm 0.099 \\
\hline 12,28 & $\mp 0.205$ & 0.281 & $\mp 0.344$ & -0.194 & \pm 0.017 & -0.259 & \pm 0.335 \\
\hline 14,26 & $\mp 0.113$ & 0.306 & $\mp 0.312$ & 0.027 & \pm 0.279 & -0.302 & \pm 0.035 \\
\hline 16,24 & $\mp 0.056$ & 0.285 & $\mp 0.216$ & 0.223 & \pm 0.336 & -0.054 & $\mp 0.290$ \\
\hline 18,32 & $\mp 0.023$ & 0.260 & $\mp 0.107$ & 0.343 & \pm 0.214 & 0.227 & $\mp 0.288$ \\
\hline 20 & 0.000 & 0.249 & 0.000 & 0.381 & 0.000 & 0.343 & 0.000 \\
\hline $2 n \backslash k$ & 6 & 5 & 4 & 3 & 2 & 1 & 0 \\
\hline 0,40 & $\cdots$ & $\cdots$ & $\cdots$ & $\ldots$ & $\ldots$ & $\ldots$ & $\ldots$ \\
\hline 2,32 & -0.004 & $\mp 0.002$ & 0.001 & $\ldots$ & $\ldots$ & $\ldots$ & $\ldots$ \\
\hline 4,36 & 0.037 & \pm 0.020 & -0.009 & \pm 0.004 & -0.001 & $\ldots$ & $\ldots$ \\
\hline 6,34 & -0.164 & $\mp 0.102$ & 0.055 & $\mp 0.026$ & 0.010 & $\mp 0.003$ & -0.001 \\
\hline 8,32 & 0.365 & \pm 0.288 & -0.192 & \pm 0.108 & -0.050 & \pm 0.018 & 0.004 \\
\hline 10,30 & -0.303 & $\mp 0.403$ & 0.381 & $\mp 0.279$ & 0.160 & $\mp 0.070$ & -0.020 \\
\hline 12,28 & -0.179 & \pm 0.108 & -0.349 & \pm 0.422 & -0.338 & \pm 0.189 & 0.067 \\
\hline 14,26 & 0.274 & \pm 0.327 & -0.070 & $\mp 0.273$ & 0.438 & $\mp 0.359$ & -0.168 \\
\hline 16,24 & 0.261 & $\mp 0.111$ & 0.357 & $\mp 0.165$ & -0.253 & \pm 0.462 & 0.320 \\
\hline 18,22 & -0.120 & $\mp 0.336$ & 0.001 & \pm 0.360 & -0.162 & $\mp 0.342$ & -0.471 \\
\hline 20 & -0.337 & 0.000 & -0.350 & 0.000 & 0.389 & 0.000 & 0.535 \\
\hline
\end{tabular}

for the coefficients $c_{2 n}$ of the eigenstate $\check{\Psi}_{k}=\Sigma_{n} c_{2 n} \breve{\Psi}(2 n)$ expanded in terms of the adiabatic mean-field states $\check{\Psi}(2 n)$, where we have defined the differences

$$
\frac{\Delta}{\Delta n} h_{2 n} \frac{\Delta}{\Delta n} c_{2 n}=h_{2 n 2 n+2} \frac{\Delta}{\Delta n} c_{2 n+1}-h_{2 n 2 n-2} \frac{\Delta}{\Delta n} c_{2 n-1}
$$

and

$$
\frac{\Delta}{\Delta n} c_{2 n+1}=c_{2 n+2}-c_{2 n}
$$

The nondiagonal elements $h$ of the Hamiltonian matrix $H$ play the role of the factor $-1 /(2 m)$ with mass $m$ in the kinetic energy term in Schrödinger equation. The negative $h$ 's in the present model are equivalent to a positive mass $m$.

It may be interesting to see in Tables I and II that higher energy states on the repulsive parabolic potential $\varepsilon_{n}=$ $-\left(g_{1}^{2} / \varepsilon\right)(2 n-N)^{2}$ in Schrödinger difference equation on the 


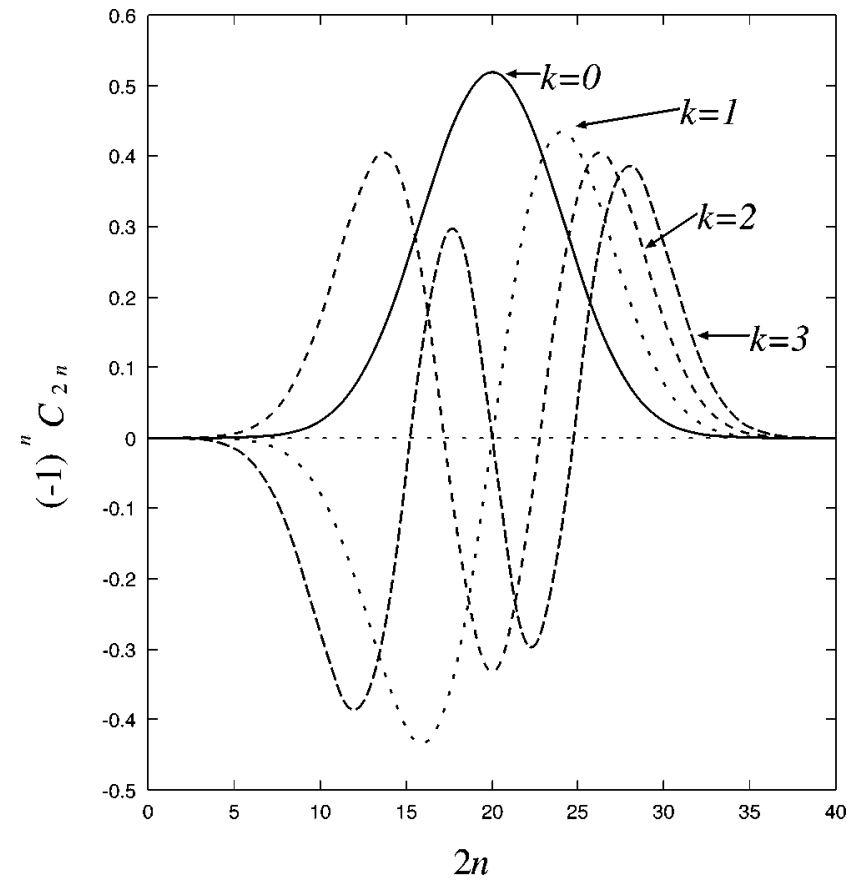

FIG. 1. The calculated distribution $(-1)^{n} c_{2 n}$ of the eigenstates $\check{\Psi}_{k}=\Sigma_{n} c_{2 n} \check{\Psi}(2 n)$ for $k=0-3$ with a sign factor $(-1)^{n}$ depending on even and odd $n$. The parameters used in this calculation are shown in the text.

finite range of integral discrete coordinates have some features resembling the energy states in an attractive parabolic (harmonic oscillator) potential. Note that the energy eigenstates $\check{\Psi}_{k}$ from $k=0$ roughly up to 5 shown in the tables have features similar to the energy states of a harmonic oscillator: They have (1) equispacing eigenenergies $E_{k}$ and (2) Gaussian distribution $(-1)^{n} c_{2 n}$ of the eigenstate wave functions $\check{\Psi}_{k}=\Sigma_{n} c_{2 n} \check{\Psi}(2 n)$ on the discrete coordinate $2 n$. In Fig. 1, we show the calculated amplitudes $(-1)^{n} c_{2 n}$ of the eigenstates $\check{\Psi}_{k}$ for $k=0$ to 3 with a sign factor $(-1)^{n}$ depending on even and odd $n$, which is equivalent to changing the sign of the nondiagonal elements $h$ of the tridiagonal Hamiltonian matrix $H$. The amplitudes look very much like the harmonic oscillator wave functions. This is because the absolute values of the elements of eigenvectors of a tridiagonal matrix are not changed, even if the signs of all diagonal elements of the matrix are changed.

\section{B. Perturbation theory for almost degenerate ground states}

The two degenerate lowest mean-field states $\check{\Psi}(0)$ and $\check{\Psi}(N)$, which are separated by a potential barrier of the energies $\varepsilon_{2 n}$ of $N / 2-1$ intermediate mean-field states $\check{\Psi}(2 n)$, are coupled to each other through the intermediate states by the residual interaction in $H_{I}$. The coupling of the two states splits their degenerate mean-field energies. While one usually calculates the splitting of the energies of the two almost degenerate ground states in the WKB method using imaginary time [8], we calculated it in an exact diagonalization in the last subsection. In this subsection we apply the perturba- tion theory to calculate the degenerate mean-field energies perturbed by the residual interaction and show that the perturbation method works for the present problem, in calculation of the splitting of the two almost degenerate ground state energies, better than the WKB method.

Putting $E$ for the eigenvalues of the Hamiltonian matrix $H$ in Eq. (35) and $I$ for $N / 2+1$ by $N / 2+1$ unit matrix, we have the eigenvalue equation $\operatorname{det}(H-E I)=0$ with the notation det standing for determinant. The determinant of the tridiagonal matrix $H-E I$ may be decomposed in terms of minor determinants $\Delta(2 n, 2 m)$ which are composed of the elements from line $2 n$ and column $2 n$ to line $2 m$ and column $2 m$ of the matrix $H-E I$. Generalizing this minor determinant expression, we may notate $\operatorname{det}(H-E I)$ as $\Delta(0, N)$.

We can prove the following two formulas for the minor determinants $\Delta(2 n, 2 m)$ of a tridiagonal matrix:

$$
\begin{aligned}
& \Delta(2 n, N-2 n) \Delta(2 n+2, N-2 n-2) \\
&- \Delta(2 n, N-2 n-2) \Delta(2 n+2, N-2 n) \\
&= h_{2 n 2 n+2} h_{2 n+22 n} h_{N-2 n-2 N-2 n} h_{N-2 n N-2 n-2} \\
& \times\{\Delta(2 n+2, N-2 n-2) \Delta(2 n+4, N-2 n-4) \\
&-\Delta(2 n+2, N-2 n-4) \Delta(2 n+4, N-2 n-2)\}
\end{aligned}
$$

and

$$
\frac{\Delta(2 n, 2 m)}{\Delta(2 n+2,2 m)}=\tilde{\varepsilon}_{2 n}-E-\frac{h_{2 n 2 n+2} h_{2 n+22 n}}{\frac{\Delta(2 n+2,2 m)}{\Delta(2 n+4,2 m)}} .
$$

Using the first formula recursively, we obtain the following relation:

$$
\begin{aligned}
& \Delta(2 n, N-2 n) \Delta(2 n+2, N-2 n-2) \\
&= \Delta(2 n, N-2 n-2) \Delta(2 n+2, N-2 n) \\
& \quad-h_{2 n 2 n+2} h_{2 n+22 n+4} \cdots h_{N-2 n-2 N-2 n} \\
& \times h_{N-2 n N-2 n-2} \cdots h_{2 n+22 n} .
\end{aligned}
$$

Applying the above formula, Eq. (44), to the eigenvalue equation $\operatorname{det}(H-E I)=0$, we decompose the eigenequation

$$
\begin{aligned}
0 & =\operatorname{det}(H-E I)=\Delta(0, N) \\
& =\left\{\Delta(0, N-2) \Delta(2, N)-\Gamma_{0 N} \Gamma_{N 0}\right\} / \Delta(2, N-2) \\
& =\Delta(2, N-2)\left[\frac{\Delta(0, N-2)}{\Delta(2, N-2)} \frac{\Delta(2, N)}{\Delta(2, N-2)}-\frac{\Gamma_{0 N} \Gamma_{N 0}}{\{\Delta(2, N-2)\}^{2}}\right],
\end{aligned}
$$


where

$$
\Gamma_{0 N}=h_{02} h_{24} \cdots h_{N-2 N}, \quad \Gamma_{N 0}=h_{N N-2} h_{N-2 N-4} \cdots h_{20} .
$$

The energy eigenvalues $E$ are obtained from the second factor on the right hand side of the above equation:

$$
\frac{\Delta(0, N-2)}{\Delta(2, N-2)} \frac{\Delta(2, N)}{\Delta(2, N-2)}-\frac{\Gamma_{0 N} \Gamma_{N 0}}{\{\Delta(2, N-2)\}^{2}}=0
$$

Applying the second formula, Eq. (43), to the two fractional factors in the first term on the left hand side of the above eigenvalue equation, we obtain the following expression for the equation:

$$
\begin{aligned}
& \left\{\tilde{\varepsilon}_{0}-E-\frac{h_{02} h_{20}}{\frac{\Delta(2, N-2)}{\Delta(4, N-2)}}\right\}\left\{\tilde{\varepsilon}_{N}-E-\frac{h_{N N-2} h_{N-2 N}}{\frac{\Delta(2, N-2)}{\Delta(2, N-4)}}\right\} \\
& -\frac{\Gamma_{0 N} \Gamma_{N 0}}{\{\Delta(2, N-2)\}^{2}}=0 .
\end{aligned}
$$

Using the perturbation theory for degenerate unperturbed energies, we solve the above eigenvalue equation for $\operatorname{det}(H$ $-E I)=0$ in order to obtain the energy eigenvalues $E$ close to the degenerate lowest unperturbed mean-field energies $\varepsilon_{0}=\varepsilon_{N}$. In perturbation theory, the energy eigenvalues $E$ in the diagonal matrix elements in $\operatorname{det}(H-E I)$ except for $\tilde{\varepsilon}_{0}$ $-E$ and $\widetilde{\varepsilon}_{N}-E$ in the diagonal $(0,0)$ and $(N, N)$ elements, respectively, are replaced by an appropriate value $\tilde{\varepsilon}_{0}$ $-h_{02} h_{20} /\left(\widetilde{\varepsilon}_{2}-\tilde{\varepsilon}_{0}\right)+\cdots$ expanded up to an order necessary for the perturbation calculation. Therefore, we replace the minor determinants $\Delta(2 n, 2 m)(0<2 n, 2 m<N)$ in the above energy eigenvalue equation (48) by $\Delta_{0}(2 n, 2 m)$, where $\Delta_{0}(2 n, 2 m)$ are the minor determinants $\Delta(2 n, 2 m)$ with the energy eigenvalues $E$ replaced by the approximate value $\tilde{\varepsilon}_{0}-h_{02} h_{20} /\left(\tilde{\varepsilon}_{2}-\tilde{\varepsilon}_{0}\right)+\cdots$. Thus, the eigenvalue equation (48) for the two almost degenerate ground state energies leads to a quadratic equation for their perturbational energy eigenvalues $E$,

$$
\begin{gathered}
\left\{\tilde{\varepsilon}_{0}-E-\frac{h_{02} h_{20}}{\left.\frac{\Delta_{0}(2, N-2)}{\Delta_{0}(4, N-2)}\right\}\left\{\tilde{\varepsilon}_{N}-E-\frac{h_{N N-2} h_{N-2 N}}{\frac{\Delta_{0}(2, N-2)}{\Delta_{0}(2, N-4)}}\right\}}\right. \\
-\frac{\Gamma_{0 N} \Gamma_{N 0}}{\left\{\Delta_{0}(2, N-2)\right\}^{2}}=0,
\end{gathered}
$$

which is equivalent to the eigenvalue equation for the two by two Hamiltonian matrix

$$
H^{\prime}=\left(\begin{array}{cc}
\tilde{\varepsilon}_{0}-\frac{h_{02} h_{20}}{\frac{\Delta_{0}(2, N-2)}{\Delta_{0}(4, N-2)}} & \frac{\Gamma_{0 N}}{\Delta_{0}(2, N-2)} \\
\frac{\Gamma_{N 0}}{\Delta_{0}(2, N-2)} & \tilde{\varepsilon}_{N}-\frac{h_{N N-2} h_{N-2 N}}{\frac{\Delta_{0}(2, N-2)}{\Delta_{0}(2, N-4)}}
\end{array}\right)
$$

This perturbation expression for the Hamiltonian matrix $H$ indicates that the two degenerate lowest mean-field energies $\widetilde{\varepsilon}_{0}$ and $\widetilde{\varepsilon}_{N}$ are perturbed by $H_{I}$ with an energy shift

$$
E_{\mathrm{sh}}=-\frac{h_{02} h_{20}}{\frac{\Delta_{0}(2, N-2)}{\Delta_{0}(4, N-2)}}=-\frac{h_{N N-2} h_{N-2 N}}{\frac{\Delta_{0}(2, N-2)}{\Delta_{0}(2, N-4)}}
$$

and an energy splitting

$$
E_{\mathrm{sp}}=\frac{2 \Gamma_{0 N}}{\Delta_{0}(2, N-2)} .
$$

The latter shows that the energy splitting is twice the perturbational energy for a hopping process on a direct path from the mean-field state $\check{\Psi}(0)$ to the degenerate counterpart $\check{\Psi}(N)$ through the intermediate mean-field states $\check{\Psi}(2 n)$ for $2 n=2,4, \ldots, N-2$. Collective tunneling takes place as a quantum mechanical hopping process along a series of intermediate states. We see that the tridiagonal mean-field Hamiltonian matrix $H$ in the present principal steering field approximation has a coherent structure in favor of dynamical collective state transitions from the state $\check{\Psi}(0)$ to $\check{\Psi}(N)$.

Assuming that the nondiagonal elements of the Hamiltonian matrix $H$ are smaller than the diagonal elements, we expand the shift $E_{\mathrm{sh}}$ and splitting $E_{\mathrm{sp}}$ of the two almost degenerate ground state energies in terms of the nondiagonal elements. Then up to second order of $H_{I}$ higher than the lowest order contribution we obtain the expression for the shift,

$$
E_{\mathrm{sh}}=-\frac{h_{02} h_{20}}{\left(\tilde{\varepsilon}_{2}-\frac{h_{24} h_{42}}{\widetilde{\varepsilon}_{4}-\widetilde{\varepsilon}_{0}}\right)-\left(\tilde{\varepsilon}_{0}-\frac{h_{02} h_{20}}{\tilde{\varepsilon}_{2}-\widetilde{\varepsilon}_{0}}\right)},
$$

and that for the splitting,

$$
E_{\mathrm{sp}}=\frac{2 \Gamma_{0 N}}{\Delta_{0}(2, N-2)},
$$

where 


$$
\begin{aligned}
& \Delta_{0}(2, N-2)=\Delta_{0}(4, N-4)\left[\frac{\Delta_{0}(2, N-4)}{\Delta_{0}(4, N-4)} \frac{\Delta_{0}(4, N-2)}{\Delta_{0}(4, N-4)}-\frac{h_{24} h_{46} \cdots h_{N-4 N-2} h_{N-2 N-4} \cdots h_{42}}{\left\{\Delta_{0}(4, N-4)\right\}^{2}}\right] \\
& =\left\{\left(\tilde{\varepsilon}_{2}-\frac{h_{24} h_{42}}{\widetilde{\varepsilon}_{4}-\tilde{\varepsilon}_{0}}\right)-\left(\tilde{\varepsilon}_{0}-\frac{h_{02} h_{20}}{\widetilde{\varepsilon}_{2}-\tilde{\varepsilon}_{0}}\right)\right\}\left\{\left(\tilde{\varepsilon}_{4}-\frac{h_{46} h_{64}}{\widetilde{\varepsilon}_{6}-\tilde{\varepsilon}_{0}}\right)-\left(\tilde{\varepsilon}_{0}-\frac{h_{02} h_{20}}{\tilde{\varepsilon}_{2}-\widetilde{\varepsilon}_{0}}\right)\right\} \ldots\left\{\left(\tilde{\varepsilon}_{N-2}-\frac{h_{N-2 N-4} h_{N-4 N-2}}{\tilde{\varepsilon}_{N-4}-\widetilde{\varepsilon}_{0}}\right)\right. \\
& \left.-\left(\tilde{\varepsilon}_{0}-\frac{h_{02} h_{20}}{\tilde{\varepsilon}_{2}-\tilde{\varepsilon}_{0}}\right)\right\}
\end{aligned}
$$

This explicit expression for the energy splitting of the two almost degenerate ground states yields an estimate of the inverse of collective tunneling time scale, as is discussed in Sec. V.

The above expressions for the shift and splitting of the two almost degenerate ground state energies indicate that when an appropriate value $\widetilde{\varepsilon}_{0}+E_{\text {sh }}$ for the eigenvalue $E$ using an approximate shift $E_{\text {sh }}$ obtained in Eq. (53) in a lower order perturbation expansion is substituted into the unperturbed energy in the expression for the splitting in Eq. (54), it accurately determines a higher order value of the small splitting of the two almost degenerate ground state energies. Table III shows that the perturbationally calculated values for the shift and splitting reproduce well the values from the exact diagonalization calculation. It shows that the present perturbation calculation works in a better precision than the WKB calculation [5].

The structure of the two by two Hamiltonian matrix $H^{\prime}$ in Eq. (50) indicates that the two degenerate lowest mean-field states $\check{\Psi}(0)$ and $\check{\Psi}(N)$ are coupled to each other through a number of intermediate mean-field states by the residual interaction in $H_{I}$ to yield a very slight splitting $E_{\mathrm{sp}}$ of the two almost degenerate ground state energies. The two by two Hamiltonian matrix $H^{\prime}$ causes the two degenerate mean-field states $\check{\Psi}(0)$ and $\check{\Psi}(N)$ to completely mix themselves in each of the two nuclear ground states:

$$
\check{\Psi}_{k}=\frac{1}{\sqrt{2}}\{\check{\Psi}(0) \pm \check{\Psi}(N)\} .
$$

The expansion coefficients $c_{2 n}$ of the two almost degenerate ground states $\check{\Psi}_{19}$ and $\check{\Psi}_{20}, \check{\Psi}_{k}=\Sigma_{n} c_{2 n} \check{\Psi}(2 n)$, in terms of

TABLE III. The shift $E_{\mathrm{sh}}$ and splitting $E_{\mathrm{sp}}$ of the energies $E_{19}$ and $E_{20}$ of the two almost degenerate ground states calculated up to first and second orders of $H_{I}$ higher than the lowest order contribution are compared with those obtained from the exact diagonalization calculation. The mean-field energies $\tilde{\varepsilon}_{0}=\tilde{\varepsilon}_{40}=$ -1.331949088 . The parameters used in this calculation are shown in the text.

\begin{tabular}{lccr}
\hline \hline Shift and splitting & First order & Second order & Exact \\
\hline$E_{\mathrm{sh}}\left(10^{-3}\right)$ & -4.44 & -4.66 & -4.68 \\
$E_{\mathrm{sp}}\left(10^{-13}\right)$ & 0.53 & 1.27 & 1.29 \\
\hline \hline
\end{tabular}

the adiabatic mean-field states $\check{\Psi}(2 n)$ exactly calculated in the diagonalization in Table II are compared with those from the perturbation calculation. We see that the two almost degenarate ground states $\check{\Psi}_{19}$ and $\check{\Psi}_{20}$ calculated in the exact diagonalization are very close to those in Eq. (55) obtained from the perturbation calculation.

\section{DYNAMICAL COLLECTIVE STATE TRANSITIONS}

In this section, we solve the time evolution problems of the nuclear system. We calculate the transition probabilities and discuss the features of dynamical collective state transitions of the system.

In terms of $N / 2+1$ adiabatic mean-field states $\check{\Psi}(2 n)$, $(2 n=0,2, \ldots, N)$, we describe the time evolution of the nuclear system. We define a $N / 2+1$ by $N / 2+1$ orthogonal matrix $U$ of the overlap integrals $\left\langle\check{\Psi}_{k} \mid \check{\Psi}(2 n)\right\rangle$ between the eigenstates $\check{\Psi}_{k}$ of $H$ and the adiabatic mean-field states $\check{\Psi}(2 n)$ with its inverse matrix $U^{-1}$. The transition amplitudes of the system from a nuclear mean-field state $\check{\Psi}(2 n)$ to another at time $t$ are expressed by $U^{-1} e^{-i H t} U$, where the time evolution matrix $e^{-i H t}$ is expressed by a diagonal matrix,

$$
e^{-i H t}=\left(\begin{array}{ccc}
e^{-i E_{0} t} & & 0 \\
& e^{-i E_{1} t} & \\
0 & \cdots & e^{-i E_{\overline{2}}^{N} t}
\end{array}\right)
$$

Let us assume that the nuclear system starts the time evolution with one of the two degenerate lowest mean-field states, $|\check{\Psi}(0)\rangle$ at time $t=0$. The nuclear state at time $t$ is

$$
|\check{\Psi}(0), t\rangle=e^{-i H t}|\check{\Psi}(0)\rangle .
$$

The transition amplitude to an adiabatic mean-field state $|\check{\Psi}(2 n)\rangle$ at time $t$ is

$$
\langle\check{\Psi}(2 n) \mid \check{\Psi}(0), t\rangle=\sum_{k=0}^{N / 2}\left\langle\check{\Psi}(2 n) \mid \check{\Psi}_{k}\right\rangle e^{-i E_{k} t}\left\langle\check{\Psi}_{k} \mid \check{\Psi}(0)\right\rangle .
$$




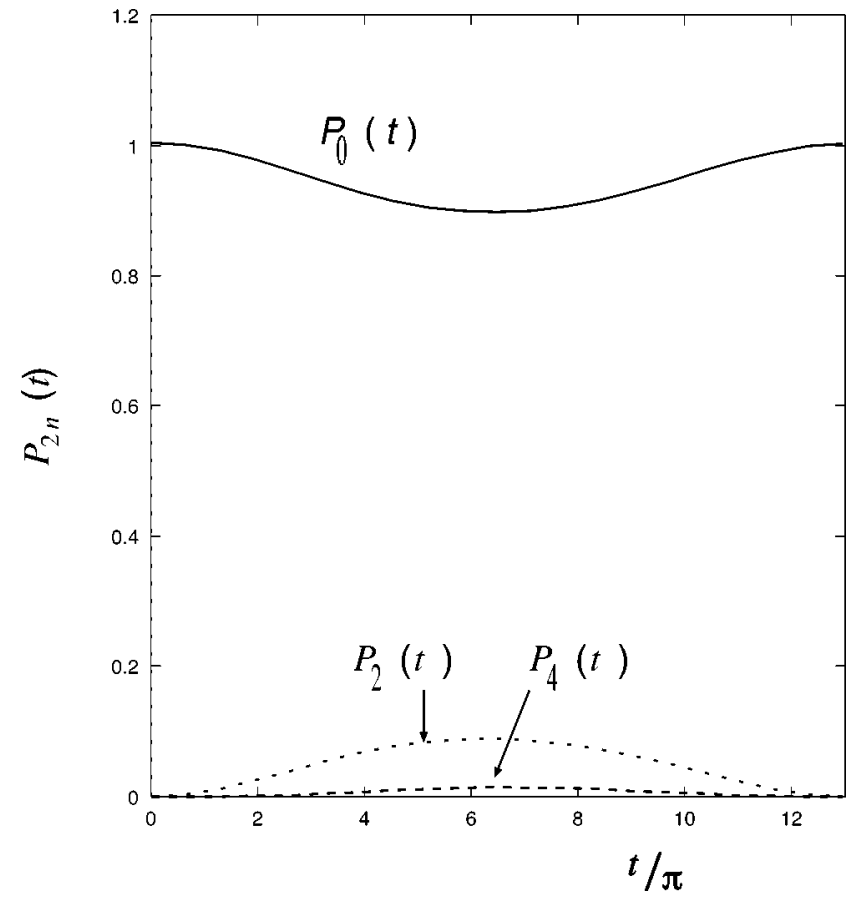

FIG. 2. The calculated transition probabilities $P_{0}(t)$ (solid line), $P_{2}(t)$ (dotted line), and $P_{4}(t)$ (dashed line) from a lowest meanfield state $\check{\Psi}(0)$ to the states $\check{\Psi}(0), \check{\Psi}(2)$, and $\check{\Psi}(4)$, respectively, in a short time evolution $0<t<2 \pi /\left(E_{18}-E_{20}\right)=13.0 \pi$. The parameters used in this calculation are shown in the text.

We see that all the eigenenergies $E_{k}$ contribute to the transition amplitude. The nuclear state at time $t$ may be expanded in terms of the nuclear mean-field states $\check{\Psi}(2 n)$,

$$
|\check{\Psi}(0), t\rangle=\sum_{n=0}^{N / 2} c_{2 n}(t)|\check{\Psi}(2 n)\rangle,
$$

with the coefficients

$$
c_{2 n}(t)=\langle\check{\Psi}(2 n) \mid \check{\Psi}(0), t\rangle .
$$

The transition probabilities to mean-field state $\check{\Psi}(2 n)$ are

$$
P_{2 n}(t)=\left|c_{2 n}(t)\right|^{2}=|\langle\check{\Psi}(2 n) \mid \check{\Psi}(0), t\rangle|^{2} .
$$

The transition amplitudes in Eq. (58) show that in a short time evolution, starting with the mean-field state $\check{\Psi}(0)$, the nuclear system oscillates quantum mechanically changing the state between the mean-field states $\check{\Psi}(0)$ and $\check{\Psi}(2)$ in a period $T=2 \pi /\left(E_{18}-E_{20}\right)$ and gradually widens the configuration space toward the mean-field states $\check{\Psi}(4), \check{\Psi}(6), \ldots$ successively. In Fig. 2, we show the calculated transition probabilities $P_{2 n}(t)$ to the adiabatic mean-field states $\check{\Psi}(2 n)$ for $2 n=0,2$, and 4 in the short period $t<2 \pi /\left(E_{18}-E_{20}\right)$ $=13.0 \pi$. The nuclear system cannot reach the degenerate counterpart $\check{\Psi}(40)$ in the short time evolution. However, in a long time evolution, the nuclear system gets to oscillate, cov-

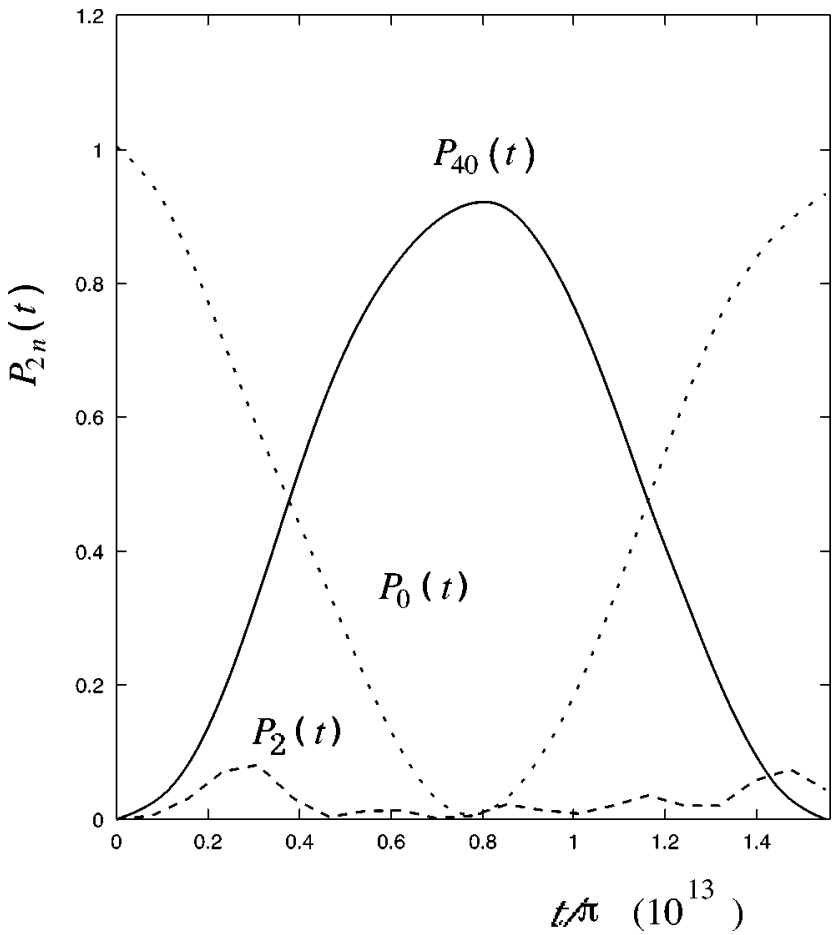

FIG. 3. The calculated transition probabilities $P_{40}(t)$ (solid line), $P_{0}(t)$ (dotted line), and $P_{2}(t)$ (dashed line) from a lowest mean-field state $\check{\Psi}(0)$ to the states $\check{\Psi}(40), \check{\Psi}(0)$, and $\check{\Psi}(2)$, respectively, in a long time evolution $0<t<2 \pi /\left(E_{19}-E_{20}\right)=1.55$ $\times 10^{13} \pi$. We use large steps of time interval of $7.8 \times 10^{11} \pi$ for the plot. If we were to take small time intervals of the order of $\pi /\left(E_{18}-E_{20}\right)=6.5 \pi$, we might see small fluctuations of the transition probabilities. The parameters used in this calculation are shown in the text.

ering the two isolated mean-field minima $\check{\Psi}(0)$ and $\check{\Psi}(40)$ in a period $T=2 \pi /\left(E_{19}-E_{20}\right)$. The transition probabilities $P_{0}(t)$ and $P_{40}(t)$ almost satisfy the unitarity, $P_{0}(t)$ $+P_{40}(t)=1$, at any time $t$. In Fig. 3, we plot the calculated transition probabilities $P_{40}(t), P_{0}(t)$, and $P_{2}(t)$ to the meanfield states $\check{\Psi}(40), \check{\Psi}(0)$, and $\check{\Psi}(2)$, respectively, in the long periodic time evolution $t<2 \pi /\left(E_{19}-E_{20}\right)=1.55$ $\times 10^{13} \pi$.

Generally speaking, a nuclear system oscillates in a number of oscillation modes with period $t=2 \pi /\left(E_{k}-E_{k^{\prime}}\right)$. When starting with the state $\Psi(0)$, however, it oscillates mainly in the two modes with the period $t=2 \pi /\left(E_{19}-E_{20}\right)$ and $t=2 \pi /\left(E_{18}-E_{20}\right)$ : the former is a time scale for tunneling and the latter is for local quantum fluctuation. Note that the transition probabilities $P_{0}(t), P_{2}(t)$, and $P_{40}(t)$ make small fluctuations in a short period $T=2 \pi /\left(E_{18}-E_{20}\right)$ even after a long time evolution. For the plot of the transition probabilities in Fig. 3, we actually use large steps of time interval of $7.8 \times 10^{12} \pi$. If we were to take small time intervals of the order of $\pi /\left(E_{18}-E_{20}\right)=6.5 \pi$ for the plot, Fig. 3 might show the small fluctuations of the transition probabilities. In Fig. 4, we show the calculated small fluctuations of the transition probabilities in the short period $T=2 \pi /\left(E_{18}\right.$ $\left.-E_{20}\right)=13.0 \pi$ after a long time evolution $t=\frac{1}{2} t_{m}$ with $t_{m}$ 


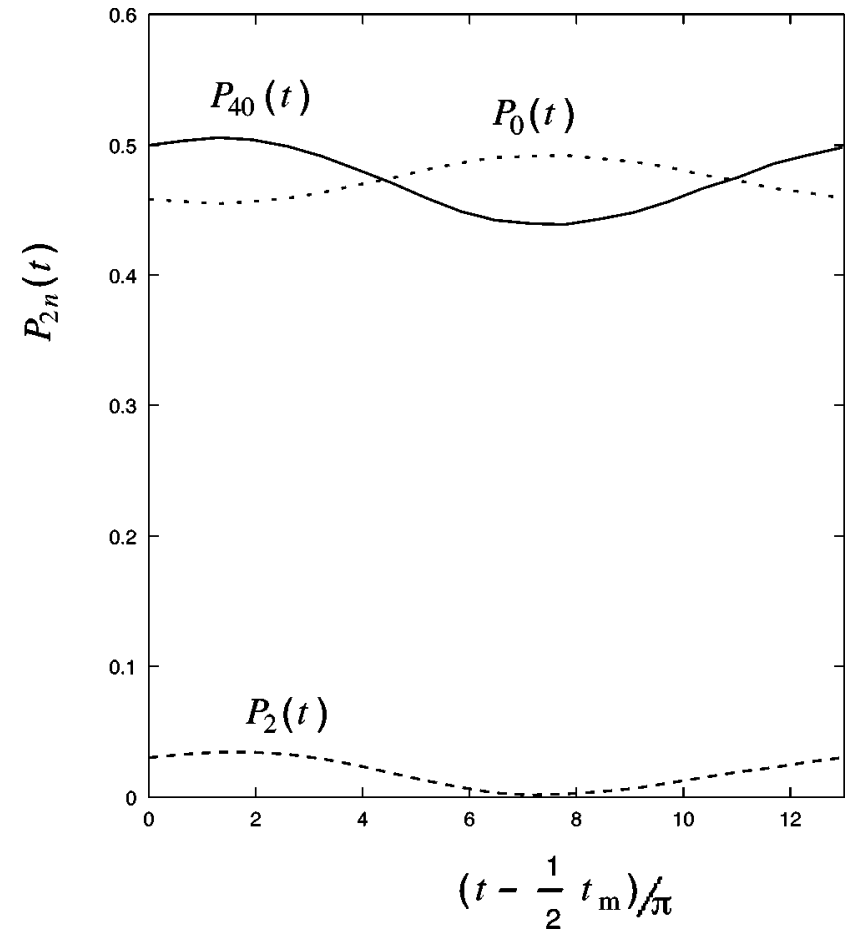

FIG. 4. The calculated fluctuating transition probabilities $P_{40}(t)$ (solid line), $P_{0}(t)$ (dotted line), and $P_{2}(t)$ (dashed line) from a lowest mean-field state $\check{\Psi}(0)$ to the states $\check{\Psi}(40), \check{\Psi}(0)$, and $\check{\Psi}(2)$, respectively, in the short period $T=2 \pi /\left(E_{18}-E_{20}\right)$ $=13.0 \pi$ after a long time evolution $t=\frac{1}{2} t_{m}$ with $t_{m}=\pi /\left(E_{19}\right.$ $\left.-E_{20}\right)=7750981924759.3 \pi$. The parameters used in this calculation are shown in the text.

$=\pi /\left(E_{19}-E_{20}\right)=7750981924759.3 \pi$, when the transition probability to the degenerate counterpart $\check{\Psi}(40)$ is predicted to be maximum. We show above the detailed value of $t_{m}$, because the transition probabilities shown in Fig. 4 are very sensitive to the value. The detail of the value is necessary to reproduce the curves.

In order to see how the nuclear state propagates from the initial mean-field state $\check{\Psi}(0)$ to the degenerate counterpart $\check{\Psi}(40)$, we show in Fig. 5 the calculated absolute values $\left|c_{2 n}(t)\right|$ of the expansion coefficients of the nuclear state $|\check{\Psi}(0), t\rangle=\Sigma_{n} c_{2 n}(t)|\check{\Psi}(2 n)\rangle$ at time $t=t_{1}=\frac{1}{4} t_{m}, t_{2}=\frac{1}{2} t_{m}$, $t_{3}=\frac{3}{4} t_{m}$, and $t_{4}=t_{m}$. The calculated values $c_{2 n}(t)$ of the coefficients show that the state amplitudes $c_{2 n}(t)$ with $2 n$ $=0$ and 40 for the mean-field states $\check{\Psi}(0)$ and $\check{\Psi}(40)$, respectively, oscillate with an amplitude close to unity but that those for the intermediate mean-field states $\check{\Psi}(2 n)$ for $n$ $=1-19$ are very small at any time, a general feature of tunneling processes. This indicates that the physical transition of the nuclear system starting with the mean-field state $\check{\Psi}(0)$ to any intermediate mean-field states $\check{\Psi}(2 n)$ for $n=1-19$ is very rare because it breaks the energy conservation.

The expansion coefficients $c_{2 n}(t)$ of the nuclear state $|\check{\Psi}(0), t\rangle=\Sigma_{n} c_{2 n}(t)|\check{\Psi}(2 n)\rangle$ satisfy the Schrödinger difference equation

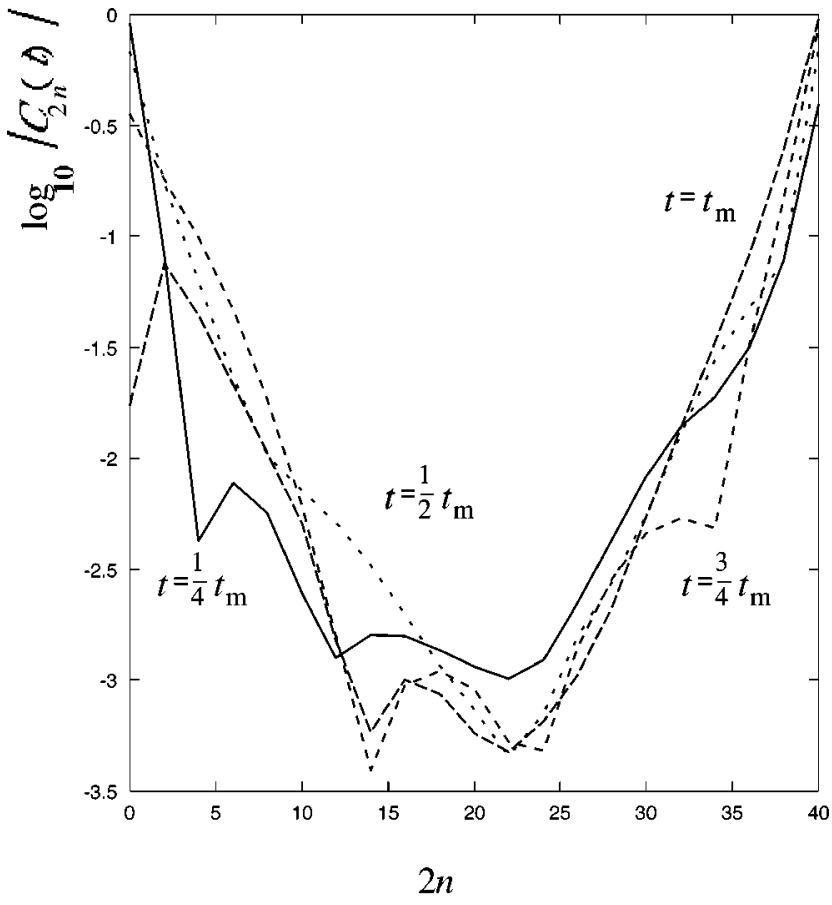

FIG. 5. The calculated logarithmic absolute values $\log _{10}\left|c_{2 n}(t)\right|$ of the expansion coefficients of the nuclear state $|\check{\Psi}(0), t\rangle$ $=\Sigma_{n} c_{2 n}(t)|\check{\Psi}(2 n)\rangle$ in terms of the mean-field states $|\check{\Psi}(2 n)\rangle$ at time $t=t_{1}=\frac{1}{4} t_{m}$ (solid line), $t_{2}=\frac{1}{2} t_{m}$ (dotted line), $t_{3}=\frac{3}{4} t_{m}$ (short dashed line), and $t_{4}=t_{m}$ (dashed line), where $t_{m}=\pi /\left(E_{19}-E_{20}\right)$ $=7750981924759.3 \pi$. The parameters used in this calculation are shown in the text.

$$
\begin{aligned}
i \frac{\partial}{\partial t} c_{2 n}(t)= & \varepsilon_{2 n} c_{2 n}(t)+\sum_{m} h_{2 n 2 m} c_{2 m}(t) \\
= & \frac{\Delta}{\Delta n} h_{2 n} \frac{\Delta}{\Delta n} c_{2 n}(t)+\left(\tilde{\varepsilon}_{2 n}+h_{2 n 2 n-2}\right. \\
& \left.+h_{2 n 2 n+2}\right) c_{2 n}(t)
\end{aligned}
$$

in the finite range of integral discrete coordinates [see Eq. (39)] with the matrix elements $h_{2 n 2 m}=\langle\check{\Psi}(2 n)|$ $H_{I}|\check{\Psi}(2 m)\rangle$ of the interaction Hamiltonian. Therefore the transition probabilities $P_{2 n}(t)=\left|c_{2 n}(t)\right|^{2}$ of the nuclear system $|\check{\Psi}(0), t\rangle$ to the mean-field state $|\check{\Psi}(2 n)\rangle$ at time $t$ satisfy the equation

$$
\begin{aligned}
\frac{\partial}{\partial t} P_{2 n}(t)= & -i \sum_{m}\left\{c_{2 n}^{*}(t) h_{2 n 2 m} c_{2 m}(t)\right. \\
& \left.-c_{2 m}^{*}(t) h_{2 m 2 n} c_{2 n}(t)\right\} .
\end{aligned}
$$

The currents for the nuclear system $|\check{\Psi}(0), t\rangle$ to change the state from the mean-field state $\check{\Psi}(2 n)$ to $\check{\Psi}(2 n+2)$ at time $t$ are defined to be 

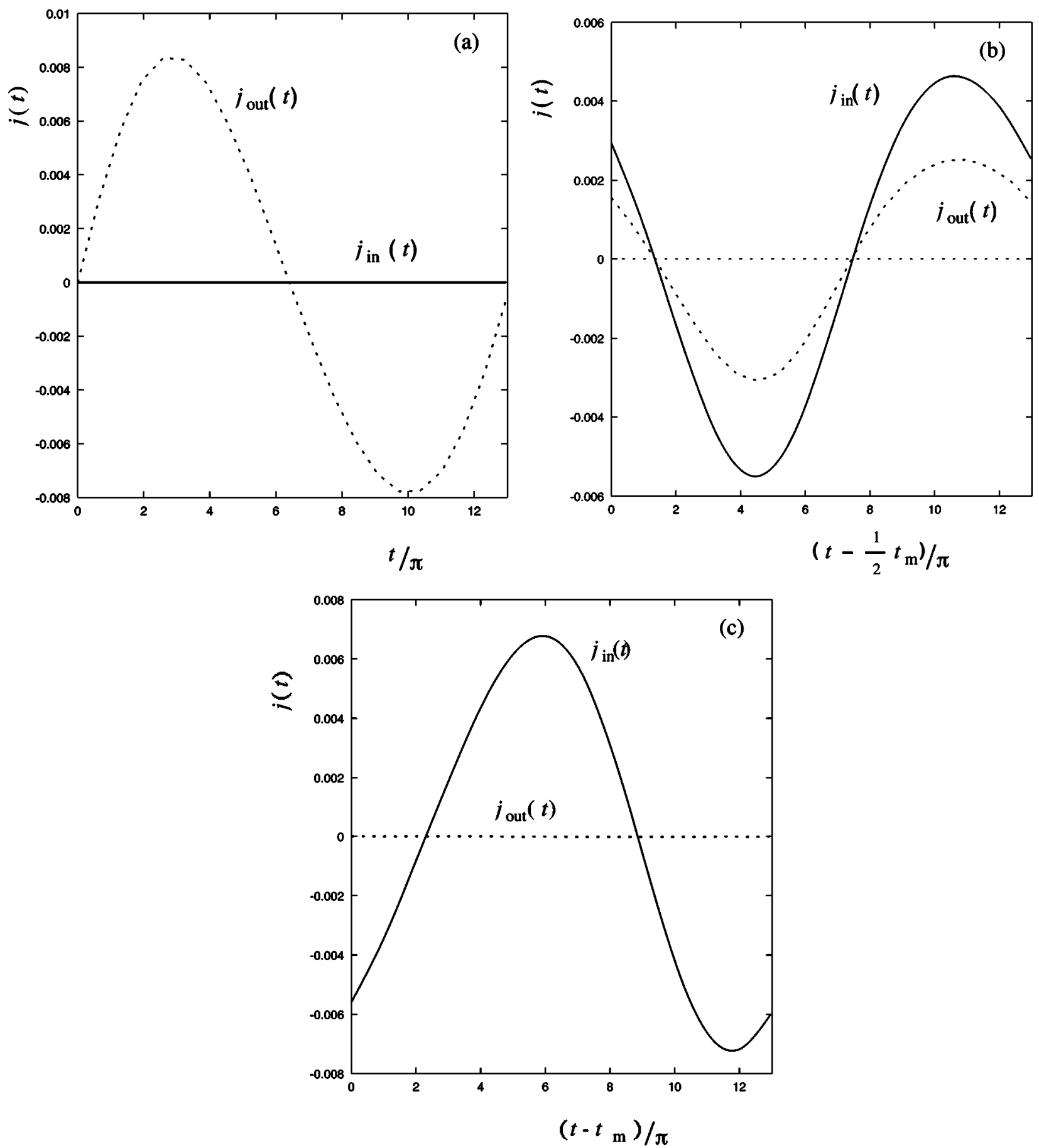

FIG. 6. The calculated fluctuating currents $j_{\text {out }}(t)$ (dotted line) and $j_{\text {in }}(t)$ (solid line), the current flowing out from the initial lowest mean-field state $\check{\Psi}(0)$ and that flowing into the degenerate counterpart $\check{\Psi}(40)$, respectively, in the short period $T=2 \pi /\left(E_{18}-E_{20}\right)$ $=13.0 \pi$ after time $t=0, \frac{1}{2} t_{m}$, and $t_{m}$, i.e., (a) $0<t<T$, (b) $\frac{1}{2} t_{m}<t<\frac{1}{2} t_{m}+T$, and (c) $t_{m}<t<t_{m}+T$, respectively, with $t_{m}=\pi /\left(E_{19}\right.$ $\left.-E_{20}\right)=7750981924759.3 \pi$. The parameters used in this calculation are shown in the text.

$$
\begin{aligned}
j_{2 n+22 n}(t)= & -i\left\{c_{2 n+2}^{*}(t) h_{2 n+22 n} c_{2 n}(t)\right. \\
& \left.-c_{2 n}^{*}(t) h_{2 n} 2 n+2 c_{2 n+2}(t)\right\},
\end{aligned}
$$

so that they satisfy the continuity equation

$$
-\frac{\partial}{\partial t} P_{2 n}(t)=j_{2 n+2} 2 n(t)-j_{2 n} 2 n-2(t)
$$

In Fig. 6, we show the calculated currents $j_{\text {out }}(t)$ $=j_{20}(t)$ and $j_{\text {in }}=j_{4038}(t)$, the current flowing out from the initial mean-field state $\check{\Psi}(0)$ and that flowing into the degenerate counterpart $\check{\Psi}(40)$, respectively, in the short period $T$ $=2 \pi /\left(E_{18}-E_{20}\right)=13.0 \pi$ after time $t=0, \frac{1}{2} t_{m}$ and $t_{m}$, i.e., (a) $0<t<T$, (b) $\frac{1}{2} t_{m}<t<\frac{1}{2} t_{m}+T$, and (c) $t_{m}<t<t_{m}+T$, respectively, with $t_{m}=\pi /\left(E_{19}-E_{20}\right)=7750981924759.3 \pi$. 


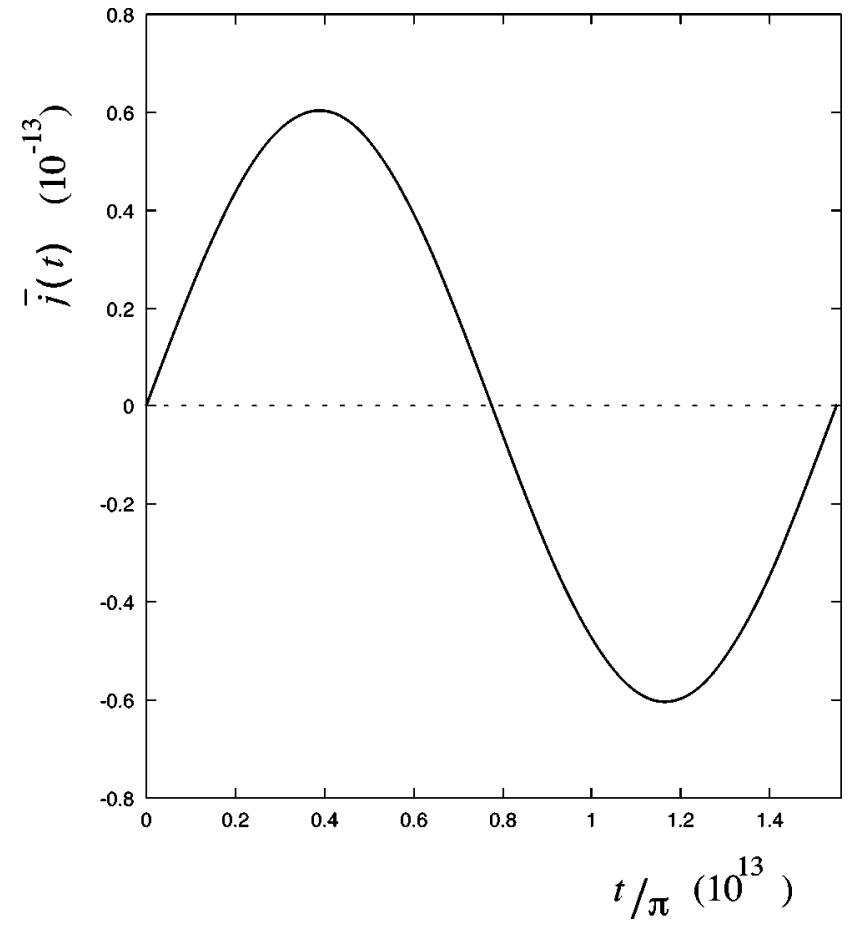

FIG. 7. The net tunneling current $\bar{j}(t)$ from the initial lowest mean-field state $\check{\Psi}(0)$ to the degenerate counterpart $\check{\Psi}(40)$ in a long time evolution $0<t<2 \pi /\left(E_{19}-E_{20}\right)=1.55 \times 10^{13} \pi$ calculated in the two-eigenenergy $\left(E_{19}\right.$ and $\left.E_{20}\right)$ approximation. The parameters used in this calculation are shown in the text.

We see that the currents $j_{\text {out }}(t)$ and $j_{\text {in }}(t)$ fluctuate quantum mechanically in the short period $T$ even after a long time evolution, except for the vanishing currents $j_{\text {in }}(t)$ in Fig. 6(a) and $j_{\text {out }}(t)$ in Fig. 6(c).

The currents $j_{2 n+22 n}(t)$ involving the quantum mechanically fluctuating local currents are very large compared with the net current for collective tunneling of the nuclear system from the initial mean-field state $\check{\Psi}(0)$ to the degenerate counterpart $\check{\Psi}(40)$ in a long time evolution. We calculate the net tunneling current $\bar{j}(t)$ in a long time evolution which is defined to be the fluctuating total currents $j(t)$ averaged in a short time evolution. For the calculation of the net tunneling current, we take into account only the two terms with $k$ $=19$ and 20 (two-eigenenergy approximation) in the transition amplitude of the nuclear state $|\check{\Psi}(0), t\rangle$ in Eq. (58), neglecting the random phase terms in a long time evolution of the system. In this approximation the net tunneling current $\bar{j}_{\text {out }}(t)$ is equal to $\bar{j}_{\text {in }}(t)$. In Fig. 7, we show the net tunneling current $\bar{j}(t)$ from the mean-field state $\check{\Psi}(0)$ to $\check{\Psi}(40)$ in a long time evolution $0<t<2 \pi /\left(E_{19}-E_{20}\right)=1.55 \times 10^{13} \pi$ calculated in the two-eigenenergy approximation. The net tunneling current $\bar{j}(t)$ of the order of $10^{-13}$ is smaller by a factor of $10^{-10}$ than the fluctuating total currents $j_{\text {out }}(t)$ and $j_{\text {in }}(t)$ of the order of $10^{-3}$. The calculated currents at the top of the potential barrier, $j_{22} 20(t)$, for example, are also fluctuating, to be of the order of $10^{-8}$ at any time $t$.

\section{CONCLUSIONS AND DISCUSSION}

We develop a fundamental description for nuclear collective transitions between Hartree minima. In our description we express nuclear metastable states in terms of the meanfield approximation. The nuclear transition from one of the metastable states to another is formulated in the steering meson field approximation. This model is more fundamental and applicable than the intuitive model of Arve et al. We can extend the present model to a more physical model, taking into account the meson fields $\varphi_{k}, k=2,3, \ldots$, in Eq. (18), orthogonal to the principal steering field $\varphi_{1}$.

Our formulation for nuclear transitions is characterized by a real time treatment instead of the usual imaginary time expression [8]. In this formulation we can describe the real time evolution of the system.

In terms of the creation and annihilation operators of nucleons and the steering field, the Hamiltonian for the nuclear system in the steering field approximation reads

$$
\begin{aligned}
H= & E\left(\alpha_{n l m}^{\mathrm{i}}\right)+\sum_{j} \varepsilon_{j}: c_{j}^{\dagger} c_{j}:+\varepsilon a_{1}^{\dagger} a_{1} \\
& -g \sum_{i j} v_{i j}: c_{i}^{\dagger} c_{j}:\left(a_{1}+a_{1}^{\dagger}\right),
\end{aligned}
$$

where the overlap of the wave functions

$$
v_{i j}=\int \psi_{i}^{*}(\vec{r}) \psi_{j}(\vec{r}) \varphi_{1}(\vec{r}) d^{3} r .
$$

In the picture of relativistic mean-field theory, the scalar $(\sigma)$ and vector $(\omega)$ meson fields produce the mean fields in the nucleus. The steering field is a linear combination of the two meson fields and here we express it to reproduce their mean-field values as

$$
\begin{gathered}
a_{1}=c_{\sigma} a_{\sigma}+c_{\omega} a_{\omega}, \\
a_{1}^{\dagger}=c_{\sigma} a_{\sigma}^{\dagger}+c_{\omega} a_{\omega}^{\dagger}, \\
a_{\sigma}=N_{\sigma} \sum_{n l m}\left(\alpha_{\sigma n l m}^{\mathrm{f}}-\alpha_{\sigma n l m}^{\mathrm{i}}\right) a_{\sigma n l m}, \\
a_{\omega}=N_{\omega} \sum_{n l m}\left(\alpha_{\omega n l m}^{\mathrm{f}}-\alpha_{\omega n l m}^{\mathrm{i}}\right) a_{\omega n l m}, \\
c_{\sigma}=\frac{\sqrt{\varepsilon_{\omega}^{3} g_{\sigma}}}{\sqrt{\varepsilon_{\sigma}^{3} g_{\omega}^{2}+\varepsilon_{\omega}^{3} g_{\sigma}^{2}}}, \\
c_{\omega}=\frac{\sqrt{\varepsilon_{\sigma}^{3} g_{\omega}}}{\sqrt{\varepsilon_{\sigma}^{3} g_{\omega}^{2}+\varepsilon_{\omega}^{3} g_{\sigma}^{2}}} .
\end{gathered}
$$

Using the best-fit values of the parameters in the relativistic mean-field models [6], we can estimate the values of the parameters in the present model. The energy $\varepsilon$ of steering field quanta is related to the energies $\varepsilon_{\sigma}$ and $\varepsilon_{\omega}$ of the scalar and vector mesons, respectively, 


$$
\varepsilon=c_{\sigma}^{2} \varepsilon_{\sigma}-c_{\omega}^{2} \varepsilon_{\omega} \simeq 100 \mathrm{MeV}
$$

and the nucleon-steering field coupling constants are

$$
\begin{gathered}
g_{1}=\left(c_{\sigma} \frac{1}{\sqrt{2 \varepsilon_{\sigma}}} g_{\sigma}-c_{\omega} \frac{1}{\sqrt{2 \varepsilon_{\omega}}} g_{\omega}\right) \frac{1}{2}\left(v_{i i}-v_{j j}\right) \simeq 1 \mathrm{MeV}, \\
g_{2}=\left(c_{\sigma} \frac{1}{\sqrt{2 \varepsilon_{\sigma}}} g_{\sigma}-c_{\omega} \frac{1}{\sqrt{2 \varepsilon_{\omega}}} g_{\omega}\right) v_{i j} \simeq 1 \mathrm{MeV}
\end{gathered}
$$

where $i$ and $j$ stand for typical particle and hole states, respectively. In the above relations for the steering field energy and the coupling constants, we have shown their typical estimated values. These meson theoretical values of $\varepsilon, g_{1}^{2}$, and $g_{2}^{2}$ are nearly one order of magnitude larger than the values of the parameters in the model of Arve et al. But it is interesting to see that the meson theoretical values of $\varepsilon, g_{1}^{2}$, and $g_{2}^{2}$ reproduce the values of the potential barrier height $\left(g_{1} N\right)^{2} / \varepsilon$ and of the residual interaction strength $g_{2}^{2} / \varepsilon$ obtained from the phenomenological observation by Arve et al. Therefore, even if we use these meson theoretical values of the parameters in the present mean- and steering-field calculation, we shall obtain the same results as the present ones.

The eigenstates in a repulsive parabolic potential for a finite dimensional matrix have similar features to those of harmonic oscillator. The amplitudes of the eigenstates in the repulsive parabolic potential in Fig. 1 look very much like harmonic oscillator wave functions. This is because the absolute values of the elements of eigenvectors of a tridiagonal matrix are not changed, even if the signs of all diagonal elements of the matrix are changed. The eigenstates of a matrix are, however, affected by the edges of the limited space for the finite dimensional matrix. Therefore the similarity of the eigenstates for a finite dimensional matrix to those of a harmonic oscillator is broken in lower energy states in the repulsive parabolic potential.

The nuclear system oscillates in a number of oscillation modes with a period of the inverse of the eigenenergy difference $E_{k}-E_{k^{\prime}}$ so that the transition probability in Eqs. (58) and (61) depends on eigenenergy differences $E_{k}-E_{k^{\prime}}$. In the case of the harmonic oscillator, the difference $E_{n}-E_{n^{\prime}}$ $=\left(n-n^{\prime}\right) \omega$ of any pair of eigenenergies $E_{n}$ and $E_{n^{\prime}}$ is an integer $n-n^{\prime}$ times the classical angular frequency $\omega$ so that the system oscillates with classical period $t=2 \pi / \omega$. In the present case of a nuclear system with a finite configuration space, a number of nonresonating frequencies $E_{k}-E_{k^{\prime}}$, the difference of eigenenergies $E_{k}$ and $E_{k^{\prime}}$, contribute to the transition. In the nuclear collective tunneling from the lowest mean-field state $\check{\Psi}(0)$ to the degenerate counterpart $\check{\Psi}(N)$, the nuclear state is dominated by lower energy eigenstates: the state is so much affected by the edges of the limited configuration space that the system does not make a harmonic oscillation. Of a number of time scales, the two time scales $t=\pi /\left(E_{19}-E_{20}\right)$ and $t=\pi /\left(E_{18}-E_{20}\right)$ concerned with the lowest energy eigenvalues are dominant: the former is an estimate of the collective tunneling time scale and the latter is of local quantum fluctuation. The system evolves oscillating mainly in the two modes with these time scales.

Our perturbation expression yields an explicit estimate, Eq. (54), for the energy splitting $E_{\mathrm{sp}}$ of the two almost degenerate ground states. This estimate of the splitting, feasible to evaluate by using physical parameters for the residual interaction and for the unperturbed energies, determines a time scale $t_{m}=\pi /\left(E_{19}-E_{20}\right)=\pi / E_{\mathrm{sp}}$ of tunneling or of fission for a particular system.

There are several nuclear states for a given nucleon configuration, $n$ nucleons in the state $s=1$ and the other $N-n$ nucleons in the state $s=-1$, because any combination of the nucleons can be chosen for the configuration. Since the single-particle energies of the nucleons are degenerate in the case of the present symmetric Hamiltonian $H$ with respect to nucleons, the nuclear transition proceeds through the nuclear states in Eq. (23), which are symmetric under the permutations among the nucleons, in the nuclear transition starting with the nuclear mean-field state $\check{\Psi}(0)$.

We can extend the present model to a more physical model. In a more physical model which takes nondegenerate single-particle states, several nucleon configurations for a given number of particles and holes may take part in dynamical collective state transitions. Then, the adiabatic mean-field Hamiltonian matrix $H$ for the system may be more complicated than a tridiagonal matrix.

When one takes several meson fields $\varphi_{k}$ in the quantum fluctuation field expansion, Eq. (19), it makes also a more complicated Hamiltonian matrix than a tridiagonal one. The present approximation for the nuclear collective state transitions in terms of the principal steering meson field suggests a way to solve the physical problems of the nuclear transitions. It may be instructive to note that the adiabatic mean-field Hamiltonian matrix for physical systems in a general case can be transformed into a tridiagonal matrix, which selects the optimum collective transition path between Hartree minima with the transformation matrix providing the path that a nuclear system takes in the course of a dynamical transition. This technique of making a tridiagonal matrix is widely used in the first step of the computational diagonalization calculus of symmetric matrices [11]. We will study this problem in another context.
[1] T. Marumori, T. Maskawa, F. Sakata, and A. Kuriyama, Prog. Theor. Phys. 64, 1294 (1980); F. Sakata, T. Marumori, Y. Hashimoto, Y. Yamamoto, H. Tsukuma, and K. Iwasawa, Nucl. Phys. A519, 93c (1990); F. Sakata, T. Marumori, Y. Hashimoto, H. Tsukuma, Y. Yamamoto, J. Terasaki, K. Iwasawa, and H. Itabashi, New Trends in Nuclear Collective
Dynamics, Springer Proceedings in Physics Vol. 58 (Springer, New York, 1992), p. 187.

[2] W. Nazarewicz, Nucl. Phys. A557, 489 (1993).

[3] M. Matsuo and K. Matsuyanagi, Prog. Theor. Phys. 74, 1227 (1985); 76, 93 (1986); 78, 591 (1987).

[4] Y. R. Shimizu and K. Matsuyanagi, Prog. Theor. Phys. 67, 
1637 (1982); 70, 144 (1983).

[5] P. Arve, G. F. Bertsch, J. W. Negele, and G. Puddu, Phys. Rev. C 36, 2018 (1987).

[6] T. Kohmura, T. Suzuki, M. Cauvin, M. Danos, and V. Gillet, Nucl. Phys. A449, 729 (1986); T. Nagata, A. Kato, and T. Kohmura, ibid. A601, 333 (1996).

[7] D. L. Hill and J. A. Wheeler, Phys. Rev. 89, 1102 (1953).
[8] J. W. Negele, Rev. Mod. Phys. 54, 913 (1982).

[9] J. D. Walecka, Ann. Phys. (N.Y.) 83, 491 (1974).

[10] H. J. Lipkin, Quantum Mechanics (North-Holland, Amsterdam, 1973), p. 373.

[11] G. H. Golub and C. F. Van Loan, Matrix Computations (Johns Hopkins University Press, Baltimore, 1983), Sec. 8.2. 